\title{
A second-order accurate non-intrusive staggered scheme for the interaction of ultra-lightweight rigid bodies with fluid flow
}

\author{
Chennakesava Kadapa* \\ Swansea Academy of Advanced Computing, Swansea University, SA1 8EN, United Kingdom
}

\begin{abstract}
This paper presents a staggered scheme with second-order temporal accuracy for fluid-structure interaction problems involving ultra-lightweight rigid bodies. The staggered scheme is based on the Dirichlet-Neumann coupling and is non-intrusive. First, the spectral properties of the staggered scheme are studied and also compared against the monolithic scheme using a linear model problem. Later, the suitability and effectiveness of the staggered scheme for problems involving incompressible flows and lightweight rigid solids are illustrated by using the examples of galloping of a square cylinder and lock-in of a circular cylinder for mass-ratio values as low as 0.01 . This is the first time in the literature flow-induced vibrations of rigid bodies with such low mass ratio values are successfully simulated using a staggered scheme. Two different fluid solvers are considered to illustrate the non-intrusive nature of the proposed scheme. Guidelines for choosing the relaxation parameter are also provided. With its iteration-free nature and with a single (relaxation) parameter, the proposed staggered scheme renders itself as an accurate and computationally efficient scheme for fluid-rigid body interaction problems, including those involving lightweight structures.
\end{abstract}

Keywords: Fluid-structure interaction; partitioned approach; staggered scheme; added-mass; lightweight structures

\section{Introduction}

Fluid-structure interaction (FSI) is a multiphysics phenomenon that is common in mechanical, civil, aerospace, biomedical, ocean and naval engineering. Vortex-induced vibrations (VIV) of chimneys, marine risers and submarine pipelines; galloping of transmission cables and bridge decks; wave-structure interaction of floating and fixed offshore structures; the aeroelastic flutter of aeroplane wings; and blood flow through heart valves and arteries, are a few examples of FSI problems.

Computer simulation of FSI is a complex and expensive task, and it poses numerous difficulties depending upon the complexity of the problem (in terms of problem geometry, structural deformations, topological changes and the model for fluid flow) and the type of numerical scheme used for the simulation [1-4]. The frequently encountered issues in simulating an FSI problem are those associated with (i) capturing large structural deformations and topological changes in the fluid domain and (ii) overcoming added-mass instabilities. A vast amount of research work has gone into addressing many of the issues associated with large structural deformations and topological changes by adapting the arbitrary Lagrangian-Eulerian (ALE) formulation with or without remeshing [5-9] and immersed/embedded/unfitted/fictitious-domain methods [10-27]. However, robust and accurate partitioned FSI algorithms that can successfully overcome significant added-mass instabilities are still lacking.

Depending upon whether the governing equations of the FSI problems are solved together or separately, the FSI schemes are termed as monolithic or partitioned schemes. In a monolithic FSI scheme [7, 20, 2734], the coupled nonlinear governing equations are solved together using an iterative technique, for example, the Newton-Raphson scheme. Granted that monolithic FSI schemes are devoid of added-mass instabilities, their disadvantages are due to the unavoidable requirement for computing off-diagonal (coupling) matrices and their high computational cost. The need to compute the coupling terms in the monolithic schemes becomes

\footnotetext{
${ }^{*}$ Corresponding author

Email address: c.kadapa@swansea.ac.uk (Chennakesava Kadapa)
} 
a serious bottleneck in combining various fluid and solid solvers in a non-intrusive manner; thus, diminishing their applicability to a limited set of solvers that satisfy certain requirements.

On the other hand, in the partitioned/staggered approaches for FSI, the solution for the coupled equations is obtained by solving the fluid and solid sub-problems separately, with an appropriate algorithm for field transfer between individual solvers. While the partitioned approaches involve sub-iterations at every time step, the staggered schemes do not. Therefore, partitioned/staggered schemes are not only computationally appealing because of their flexibility in combining various fluid and solid solvers, but also are significantly less expensive when compared to the monolithic FSI schemes. However, the main drawback of the partitioned/staggered schemes is their instabilities due to the added-mass effect.

The major cause of instabilities in the partitioned FSI algorithms is the added mass [35] which becomes predominant in the FSI problems involving lightweight structures, see Causin et al. [36], Conca et al. [37] and Förster et al. [38], van Brummelen [39] and Dettmer and Perić [42]. The added-mass instabilities can be characterised by the mass ratio $\left(m^{*}=\right.$ solid mass/displaced fluid mass) for rigid structures, and density ratio ( $\rho^{*}=$ solid density/fluid density) for flexible structures. The lower the value of $m^{*}$ (or $\rho^{*}$ ), the higher the added mass. The stability of a partitioned/staggered scheme is dictated by the value of $m^{*}$ (or $\rho^{*}$ ).

A wide variety of techniques have been proposed towards addressing the added-mass instabilities in the partitioned FSI algorithms. While some techniques solve the FSI problem without using sub-iterations, for example, the conventional sequential staggered (CSS) scheme [40], others use sub-iterations at each time step until a pre-defined convergence criterion is satisfied. Some noteworthy contributions on partitioned FSI schemes are due to Florentie et al. [41], Dettmer and Perić [42], Fernández and Moubachir [43], Burman and Fernández [44, 45], dos Santos et al. [46], Breue et al. [47], Badia et al. [48], Wood et al. [49], Matthies and Steindorf [50], Degroote et al. [29], Farhat et al. [51], Heil et al. [32], Guidoboni et al. [52], Jaiman et al. [53], Michler et al. [54], Causin et al. [36], van Brummelen [39, 55], Förster et al. [38], He [56, 57], He and Zhang [58], He et al. [59, 60], Idelsohn et al. [62], Banks et al. [63, 64], Bukac et al. [65, 66], and Winterstein et al. [67].

In spite of numerous contributions towards addressing the added-mass issues in partitioned/staggered FSI schemes, the accuracy and robustness of these algorithms are still open problems. Note that the majority of the research work is focussed on the FSI problems with flexible lightweight structures, and, of course, it is for all the correct reasons. Nevertheless, the literature on partitioned/staggered approaches that are accurate and robust for the simulation of FSI problems involving lightweight rigid solids is limited. To the best of author's knowledge, only Jaiman et al. [68], He [56, 57] and Banks et al. [63, 64] successfully demonstrated partitioned approaches to FSI problems involving lightweight rigid solids for which $m^{*}<1$.

With the novel nonlinear interface force correction (NIFC) scheme, Jaiman et al. [68, 105] successfully demonstrated vortex-induced vibrations of a circular cylinder for $m^{*}$ values as low as 0.26 . He [69] successfully adapted a partitioned implicit coupling using a displacement-predictor to successfully simulate vortex-induced vibrations of a circular cylinder for $m^{*} \geq 0.298$. Banks et al. [63, 64] proposed the added-mass partition (AMP) algorithm that can cope with even zero mass-ratio value. Despite its second-order accuracy, the NIFC scheme [68] requires sub-iterations, and the overall accuracy of the partitioned approach followed by He [69] is first-order accurate because the accuracy of the mesh update procedure is only of order one. While the AMP scheme can accommodate even zero mass-ratio value, the need to evaluate the surface integrals for computing the added-mass and added-damping tensors somewhat limits the applicability of the scheme to simplistic geometries. Moreover, the added-mass and added-damping tensors proposed in [63, 64] modify the dynamic characteristics of the original solid sub-system. Due to the lack of examples involving vortex-induced vibrations of rigid solids in [63, 64], the effect of the added-mass and added-damping tensors on the amplitude and frequency of oscillations is unknown.

To perform accurate and computationally efficient simulations of the FSI problems involving lightweight rigid solids by overcoming the issues mentioned above, we present a new staggered scheme for fluid-rigid body interaction that (i) is second-order accurate in time, (ii) involves no sub-iterations at every time step, (iii) requires only Dirichlet boundary conditions for the fluid problem and Neumann boundary conditions for the solid problem and (v) can be used to develop an FSI framework by coupling fluid and solid solvers in a nonintrusive manner. The proposed staggered scheme is an extension of the scheme proposed by Dettmer and Perić [42] to FSI problems with lightweight rigid solids. For the sake of simplicity, and for future extensions to fluidstructure-control interaction problems, the staggered scheme is reformulated using a single time integration scheme for both the fluid and solid problems. The accuracy of the staggered scheme is demonstrated using the 
examples of flow-induced vibrations involving rigid solids with mass ratio values as low as 0.01 . To the best of author's knowledge, this is the first time in the literature that successful numerical solutions are computed using a staggered scheme for fluid-rigid body interaction problems with such low $m^{*}$ values. The non-intrusive nature of the proposed scheme is illustrated using two different fluid solvers.

The rest of the paper is organised as follows. The governing equations for the fluid-rigid body interaction problems are presented in Section 2, followed by the discussion of monolithic and partitioned solution approaches to FSI problems in Section 3. The accuracy and stability of the staggered scheme are assessed by studying the single-degree-of-freedom linear spring-mass-damper system in Section 4 . Numerical damping characteristics and condition for the stability of the staggered scheme are also established in Section 4 . The remarkable ability of the staggered scheme in computing accurate numerical solutions of FSI problems involving lightweight rigid solids and its ability to combine different solid and fluid solvers in a non-intrusive manner are demonstrated using the example of vortex-induced vibrations of a circular cylinder, galloping of a square body and lightweight rectangular body rising in a liquid, in Section 5 Guidelines for choosing the relaxation parameter are outlined in Section 6. The paper is concluded with Section 7 by providing a summary of key observations made and conclusions drawn.

\section{Governing equations of the fluid-rigid body interaction problem}

This section provides a brief summary of the governing equations for the fluid-structure interaction problems involving isothermal, laminar, viscous, incompressible flows and rigid body dynamics. The domains of the fluid and solid problems are denoted as $\Omega^{f}$ and $\Omega^{s}$, respectively, with $\Gamma^{f}$ and $\Gamma^{s}$ as their respective boundaries. The interface between the fluid and solid domains is denoted as $\Gamma^{f-s}$.

\subsection{Governing equations for the fluid flow problem}

For isothermal, laminar, viscous and incompressible flows, the initial-boundary value problem is stated as: Find velocity, $\mathbf{v}^{f}: \Omega^{f} \rightarrow \mathbb{R}^{3}$; and pressure, $p^{f}: \Omega^{f} \rightarrow \mathbb{R}$, for the given $\mathbf{f}^{f}: \Omega^{f} \rightarrow \mathbb{R}^{3} ; \overline{\mathbf{v}}^{f}: \Gamma_{D}^{f} \rightarrow \mathbb{R}^{3}$; and $\overline{\mathbf{t}}^{f}: \Gamma_{N}^{f} \rightarrow \mathbb{R}^{3}$, such that:

$$
\begin{aligned}
& \rho^{f} \frac{\partial \mathbf{v}^{f}}{\partial t}+\rho^{f}\left(\mathbf{v}^{f} \cdot \nabla\right) \mathbf{v}^{f}-\mu^{f} \Delta \mathbf{v}^{f}+\nabla p^{f}=\mathbf{f}^{f} \quad \text { in } \Omega^{f}, \\
& \boldsymbol{\nabla} \cdot \mathbf{v}^{f}=0 \quad \text { in } \Omega^{f}, \\
& \mathbf{v}^{f}=\overline{\mathbf{v}}^{f} \quad \text { on } \Gamma_{D}^{f}, \\
& \boldsymbol{\sigma}^{f} \cdot \mathbf{n}^{f}=\overline{\mathbf{t}}^{f} \quad \text { in } \Gamma_{N}^{f}, \\
& \mathbf{v}^{f}(t=0)=\mathbf{v}_{0}^{f} \quad \text { in } \Omega^{f},
\end{aligned}
$$

where, $t$ is the time variable, $\nabla$ is the gradient operator, $\mu^{f}$ is the dynamic viscosity of the fluid, $\rho^{f}$ is the density of the fluid, $\mathbf{f}^{f}$ is the body force, $\mathbf{n}^{f}$ is the unit outward normal on the boundary $\Gamma^{f}, \mathbf{v}_{0}^{f}$ is the initial velocity of the fluid in the domain, $\overline{\mathbf{v}}^{f}$ is the prescribed velocity on Dirichlet boundary $\Gamma_{D}^{f}$, and $\overline{\mathbf{t}}^{f}$ is applied traction on the Neumann boundary $\Gamma_{N}^{f}$. The conditions $\Gamma^{f}=\Gamma_{D}^{f} \cup \Gamma_{N}^{f}$ and $\Gamma_{D}^{f} \cap \Gamma_{N}^{f}=\emptyset$, apply for the Dirichlet and Neumann boundaries. The pseudo-stress $\sigma^{f}$ is defined as,

$$
\boldsymbol{\sigma}^{f}:=\mu^{f} \nabla \mathbf{v}^{f}-p^{f} \mathbf{I},
$$

where, $\mathbf{I}$ is the second-order identity tensor.

\subsection{Governing equations for rigid body dynamics}

The equations governing the dynamics of rigid solids can be written as

$$
\begin{aligned}
\mathbf{M} \mathbf{a}^{s}+\mathbf{C v}^{s}+\mathbf{K} \mathbf{d}^{s} & =\mathbf{F}^{s} \\
\mathbf{d}^{s}(t=0) & =\mathbf{d}_{0}^{s}, \\
\mathbf{v}^{s}(t=0) & =\mathbf{v}_{0}^{s},
\end{aligned}
$$


where, $\mathbf{M}$ is the mass matrix, $\mathbf{C}$ is the damping matrix, $\mathbf{K}$ is the stiffness matrix, $\mathbf{d}^{s}$ is the displacement vector of the rigid body, $\mathbf{v}^{s}\left(=\mathrm{d} \mathbf{d}^{s} / \mathrm{d} t\right)$ is the velocity vector and $\mathbf{a}^{s}\left(=\mathrm{d}^{2} \mathbf{d}^{s} / \mathrm{d} t^{2}\right)$ is the acceleration vector, $\mathbf{d}_{0}^{s}$ is the initial displacement vector, $\mathbf{v}_{0}^{s}$ is the initial velocity vector and $\mathbf{F}^{s}$ is the force vector. Note that only translational degrees of freedom (DOFs) are considered in this work for the sake of simplicity. The inclusion of rotational DOFs is straightforward, of course, with appropriate modifications.

\subsection{Governing equations at the fluid-solid interface}

At the interface $\Gamma^{f-s}$ between the fluid and solid domains, the kinematic constraint and the equilibrium of tractions need to be satisfied by the solution variables in the fluid and solid domains. These two conditions can be written as,

$$
\begin{array}{rlr}
\text { Kinematic constraint: } & \mathbf{v}^{f}=\mathbf{v}^{s}, \\
\text { Traction equilibrium: } & \boldsymbol{\sigma}^{f} \cdot \mathbf{n}^{f}+\boldsymbol{\sigma}^{s} \cdot \mathbf{n}^{s}=\mathbf{0},
\end{array}
$$

where, $\mathbf{n}^{s}$ is the unit outward normal to the boundary of the solid domain. At the fluid-solid interface, we have, $\mathbf{n}^{s}=-\mathbf{n}^{f}$. The traction equilibrium (5) can be transformed into the following force equilibrium condition for the rigid-body interaction problem,

$$
\mathbf{F}^{f}+\mathbf{F}^{s}=\mathbf{0}
$$

where,

$$
\mathbf{F}^{f}=\int_{\Gamma^{f-s}} \boldsymbol{\sigma}^{f} \cdot \mathbf{n}^{f} \mathrm{~d} \Gamma
$$

\section{Formulation}

Several numerical formulations exist in the literature for solving the fluid flow problem over moving solids. Such schemes can be broadly classified into two categories: (i) Arbitrary Lagrangian-Eulerian (ALE) based methods using body-fitted meshes [5, -9,70, 71] and (ii) immersed/embedded/unfitted/CutFEM/fictitiousdomain methods [10-27]. The specific details of the formulation used for the flow problem are not particularly important in the context of the present work since the motivation behind the proposed staggered scheme is to be able to develop FSI solvers by coupling different fluid and solid sub-solvers in a non-intrusive fashion. The only requirement is that the fluid solver treats the interface velocity as the Dirichlet boundary condition and the solid solver treats the interface traction as the Neumann boundary condition, which is quite easily satisfied by the majority of opensource as well as commercial computational fluid dynamics (CFD) and rigid body dynamics solvers.

After selecting the appropriate fluid and solid solvers, the coupled FSI problem given by the equations (1)(5) can be solved using either a monolithic or a partitioned approach, as discussed in the following subsections.

\subsection{Monolithic approach}

In the monolithic approach, the coupled governing equations (1)-(5) are solved together for the solution field, typically $\left\{\mathbf{v}^{f}, p^{f}, \mathbf{v}^{s}\right\}$, using an iterative procedure such as the Newton-Raphson scheme. After adapting a spatial discretisation technique and appropriate time integration schemes for the fluid and solid sub-problems, the coupled FSI problem can be written in the matrix-vector form,

$$
\left[\begin{array}{ll}
\mathbf{K}^{f f} & \mathbf{K}^{f s} \\
\mathbf{K}^{s f} & \mathbf{K}^{s s}
\end{array}\right]\left\{\begin{array}{l}
\Delta \mathbf{V}^{f} \\
\Delta \mathbf{V}^{s}
\end{array}\right\}=-\left\{\begin{array}{l}
\mathbf{R}^{f} \\
\mathbf{R}^{s}
\end{array}\right\}
$$

where, $\mathbf{V}^{f}$ is the vector of DOFs, $\mathbf{K}^{f f}$ is the effective stiffness matrix and $\mathbf{R}^{f}$ is the residual vector, for the fluid sub-problem. $\mathbf{V}^{s}, \mathbf{K}^{s s}$ and $\mathbf{R}^{f}$ are the corresponding entries for the solid sub-problem. $\mathbf{K}^{f s}$ and $\mathbf{K}^{s f}$ are the coupling matrices which are the result of linearisation of the interface terms. The specific details of the 
individual matrices in the coupled matrix system (8) are not important, except the fact that the computation of coupling terms, $\mathbf{K}^{f s}$ and $\mathbf{K}^{s f}$, is essential for the success of the monolithic scheme.

The unique advantage of the monolithic scheme is in its ability to accommodate any mass-ratio $\left(m^{*}\right)$ values, including $m^{*}=0$. However, in addition to being computationally expensive, monolithic schemes are known to pose serious challenges in their computer implementation, especially in computing the coupling terms as well as achieving good scalability on high-performance computing architectures. Albeit the coupling terms can be computed in the in-house codes for CFD solvers based on body-fitted meshes, this task is not without its programming difficulties for complex geometries. Moreover, CFD solvers based on cut-cell methods [22] pose additional challenges in computing the coupling terms due to activation/deactivation of fluid cells from one iteration to the other. Furthermore, it is almost impossible to compute the coupling terms for combining various fluid and solid solvers that adapt different modelling techniques, for example, finite volume method (FVM) for the fluid problem and finite element method (FEM) for the solid problem. Therefore, to achieve flexibility in combining various fluid and solid solvers and solve the two-way FSI problem in a computationally efficient manner, partitioned approaches are the preferred choice.

\subsection{Partitioned or staggered approach}

In the partitioned approaches for FSI problems, the solid and fluid sub-systems are solved separately in an iterative manner until a predefined convergence criterion is satisfied. In contrast, staggered FSI schemes do not involve sub-iterations [40]. Several possibilities exist in the partitioned approaches for FSI problems; some schemes approximate the coupled matrices or use quasi-Newton methods with fixed-point iterations to achieve convergence [29], and others ignore coupled matrices altogether. Schemes based on inexact Jacobians or quasi-Newton methods are still computationally expensive and pose several challenges in parallelisation for the high-performance computing architectures [72, 73]. In the explicit coupling approaches, for example, the conventional sequentially staggered scheme (CSS) [40] and other similar variations, the fluid and solid problems are solved separately without considering the effects of coupling matrices. This feature is what makes the explicit coupling approaches computationally very appealing because such an approach is relatively easy to implement by developing wrappers for combining various fluid and solid solvers. Nevertheless, the partitioned approaches for FSI problems are not without their drawbacks, among which the instability due to added-mass is the most prominent one.

A partitioned or staggered scheme can be designed using different approaches. The majority of the partitioned schemes available in the literature are based on displacement predictors for the solid problem [38, 51, 69, 74]. Staggered schemes based on force-predictors for the solid problem also exist [42, 61, 75, 106]. Other variations in partitioned schemes include added-mass partitioned (AMP) scheme by Bank et al. [63, 64], combined interface boundary condition by Jaiman et al. [53], and nonlinear interface force correction(NIFC) by Jaiman et al. [68].

The staggered scheme considered in the present work is based on the force predictor for the solid problem. The coupling type employed is the Dirichlet-Neumann coupling in which the interface traction from the fluid solver is applied as a Neumann boundary condition for the solid domain, and the interface velocity is imposed as a Dirichlet boundary condition for the fluid problem. Dirichlet-Neumann coupling is the most intuitive type of coupling because of the nature of applied boundary conditions on the fluid and solid problems. Following the terminology of Felippa et al. [40], the temporal flow diagram for the proposed staggered scheme is depicted in Fig. 1] and the corresponding pseudocode is presented in Fig. 2. 


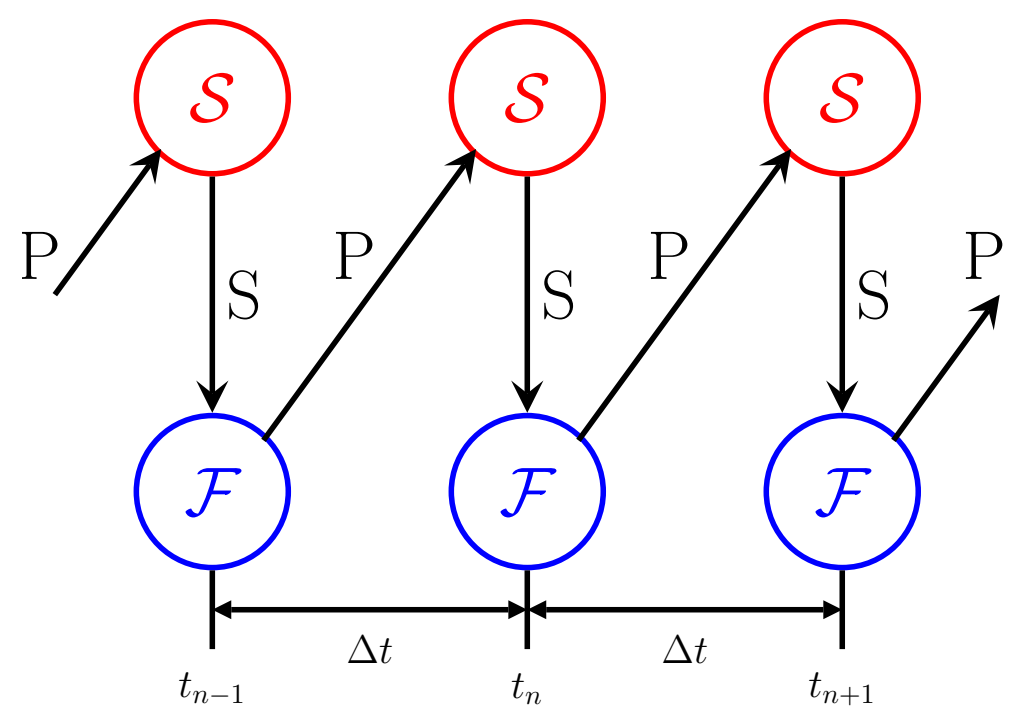

Figure 1: The temporal flow diagram for proposed staggered scheme: $\mathcal{S}$ is the solid problem, $\mathcal{F}$ is the fluid problem, $\mathrm{P}$ is the predictor step, and $\mathrm{S}$ is the substitution step. $t_{n-1}, t_{n}$ and $t_{n+1}$ are the time instants, and $\Delta t$ is the time step.

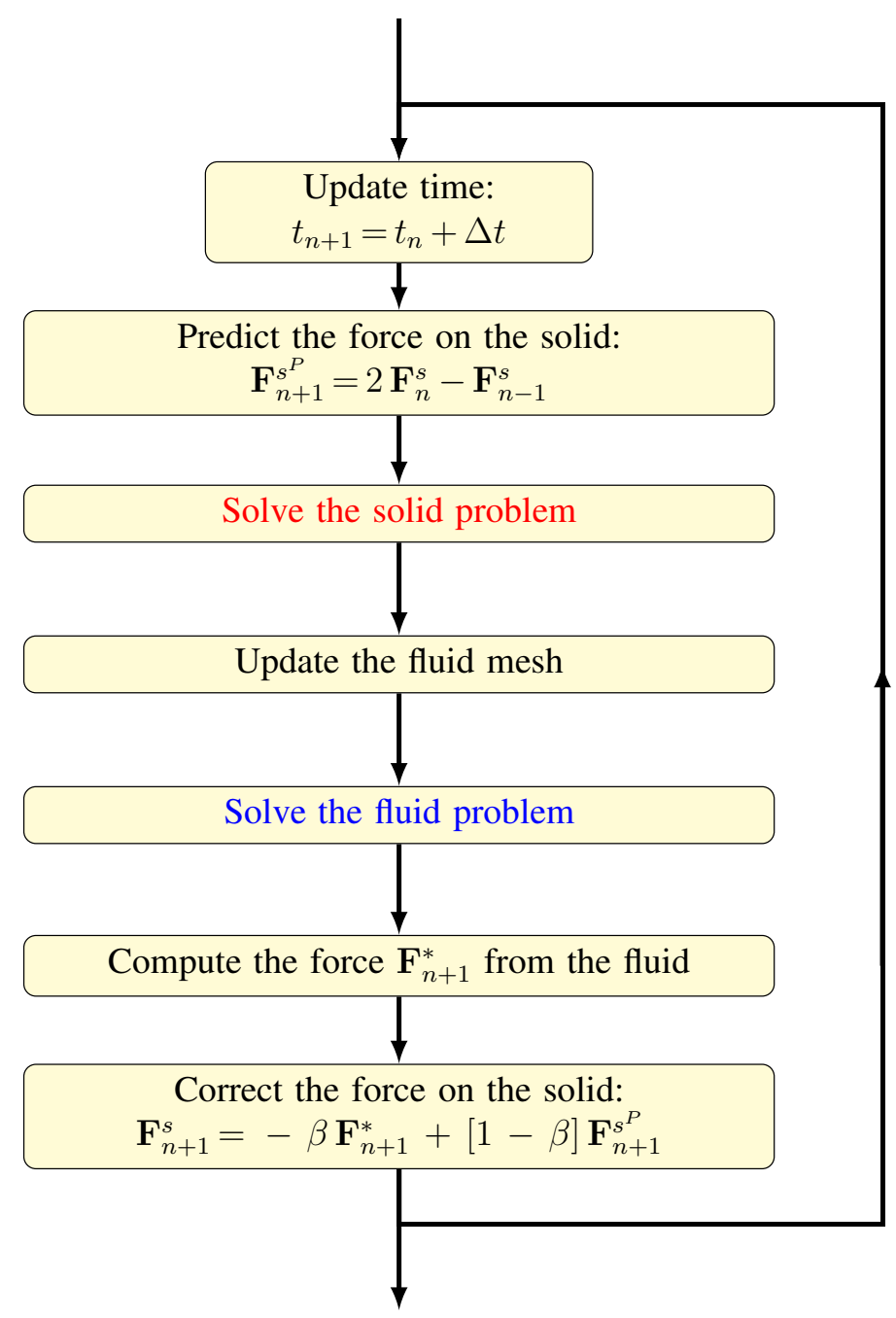

Figure 2: Key steps in the staggered scheme. Note that the scheme involves no sub-iterations at each time step. 


\section{Model problem}

Analysis of a staggered scheme for the FSI problems is quite a challenging task under the general settings of incompressible Navier-Stokes equations. Therefore, in this work, the accuracy and stability characteristics of the staggered scheme are assessed using the single-degree-of-freedom linear spring-mass-damper system and the observations are extended to the FSI problems involving actual CFD solvers. Despite its simplicity, the linear spring-mass-damper system provides a reasonable framework for conducting the spectral analysis of the staggered scheme.

The original linear problem consisting of a spring-mass-damper system is shown in Fig. 3(a), and it is modified into the one with two masses, as shown in Fig. 3(b), by distributing the mass $m$ to the solid (spring) and the fluid (damper) sub-systems such that

$$
m^{s}=\alpha m ; \quad m^{f}=[1-\alpha] m ; \quad \text { and } \quad m^{s}+m^{f}=m,
$$

where, $m^{s}$ the mass of the solid sub-system and $m^{f}$ is the mass of the fluid sub-system. The parameter $\alpha$ gives an indication of the solid mass with respect to the total mass, and it is a crucial parameter in the study of the staggered scheme. The parameter $\alpha$ is related to the mass-ratio parameter $\left(m^{*}=m^{s} / m^{f}\right)$ which is well-known in the literature on fluid-rigid solid interaction [76] as,

$$
\alpha=\frac{m^{s}}{m}=\frac{m^{*}}{1+m^{*}}
$$

The parameter $\alpha$ lies in the range $[0,1]$ for any positive value of $m^{*}$ and it approaches zero as $m^{*}$ approaches zero, as shown in Fig. 4.

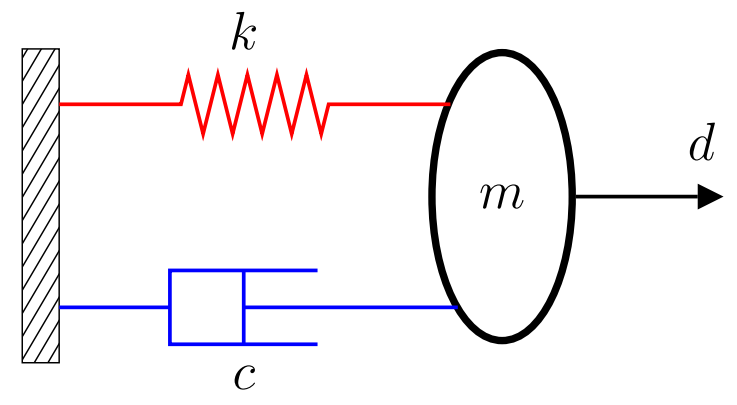

(a) Standard spring-mass-damper model

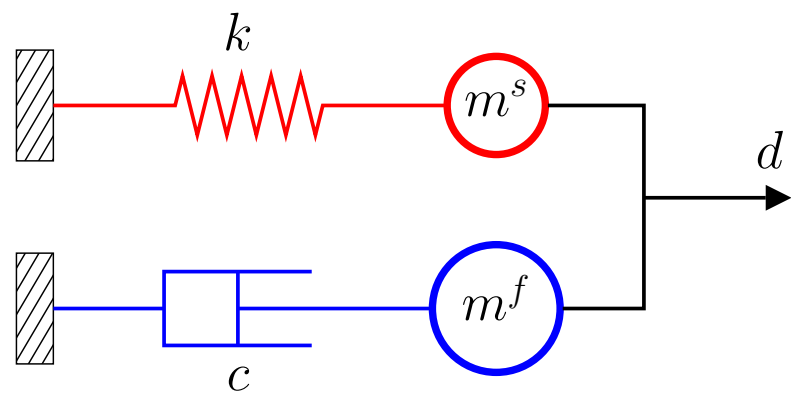

(b) Modified spring-mass-damper model

Figure 3: Linear model problem.

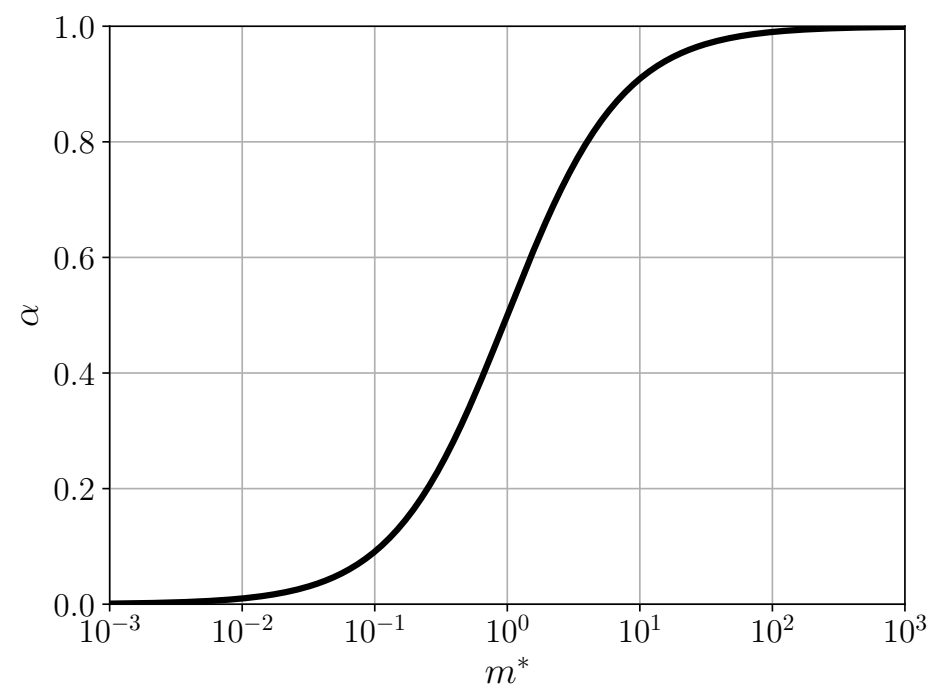

Figure 4: Variation of $\alpha$ with respect to $m^{*}$. 


\subsection{Governing equations}

Under the absence of external force, the governing equations for the original spring-mass-damper model are given by,

$$
\begin{aligned}
m \ddot{d}+c \dot{d}+k d & =0, \\
d(t=0) & =d_{0}, \\
\dot{d}(t=0) & =\dot{d}_{0},
\end{aligned}
$$

where, $m$ is the mass, $c$ is the damping coefficient, $k$ is the stiffness, $d$ is the displacement, $\dot{d}\left(=\frac{\mathrm{d} d}{\mathrm{~d} t}\right)$ is the velocity and $\ddot{d}\left(=\frac{\mathrm{d}^{2} d}{\mathrm{~d} t^{2}}\right)$ is the acceleration, $d_{0}$ is the initial displacement, and $\dot{d}_{0}$ is the initial velocity. Other useful quantities, namely, the undamped natural frequency, $\omega$, the damping ratio, $\xi$, and time period, $T$ are defined as

$$
\omega:=\sqrt{\frac{k}{m}} ; \quad \xi:=\frac{c}{2 \sqrt{k m}} ; \quad T:=\frac{2 \pi}{\omega} .
$$

The governing equations for the modified model problem can be written as,

$$
\begin{aligned}
\alpha \ddot{d}^{s}+\omega^{2} d^{s} & =f^{s}, \\
(1-\alpha) \dot{v}^{f}+2 \xi \omega v^{f} & =f^{f}, \\
\dot{d}^{s} & =v^{f}, \\
f^{s}+f^{f} & =0,
\end{aligned}
$$

where, $d^{s}, \dot{d}^{s}$ and $\ddot{d}$ are displacement, velocity and acceleration of the solid sub-system, respectively; $v^{f}(=$ $\left.\dot{d}^{f}\right), \dot{v}^{f}\left(=\ddot{d}^{f}\right)$ are the velocity and acceleration of the fluid sub-system, respectively. $f^{s}$ and $f^{f}$ are the forces on the solid and fluid sub-systems, respectively. The solid and fluid sub-system satisfy the kinematic constraint (15c) and the force equilibrium (15d) at the fluid-solid interface.

Remark 1: The staggered scheme originally proposed in [42] is based on the generalised-alpha scheme proposed by Chung and Hulbert [77] for second-order ordinary differential equations (ODEs) for structural dynamics. The present work uses the generalised-alpha scheme originally proposed by Jansen et al. [78] for the fluid problem (first-order ODE) and then later extended to structural dynamics in Kadapa [79]. The decision to use the time integration scheme for the first-order equations is for the ease of future extensions to fluid-structure-control interaction problems. Nevertheless, the staggered scheme can be used with any other second-order accurate time integration scheme, for example, the Newmark-beta method [80] or the HHT-alpha method [81].

Now, introducing an auxiliary variable $v^{s}=\dot{d}^{s}$, the system of equations 15 for the modified model problem, including the initial conditions, can be re-written as the following system of first-order equations,

$$
\begin{aligned}
\dot{d}^{s} & =v^{s}, \\
\alpha \dot{v}^{s}+\omega^{2} d^{s} & =f^{s}, \\
{[1-\alpha] \dot{v}^{f}+2 \xi \omega v^{f} } & =f^{f}, \\
v^{s} & =v^{f}, \\
f^{s}+f^{f} & =0, \\
d^{s}(t=0) & =d_{0}^{s}, \\
\dot{d}^{s}(t=0)=v^{s}(t=0)=v^{f}(t=0) & =\dot{d}_{0}^{s} .
\end{aligned}
$$




\subsection{Generalised-alpha time integration scheme for the first-order ODE}

For the first-order ODE,

$$
\dot{u}-\vartheta u=f,
$$

where, $u$ is the solution variable, $\vartheta$ is a constant and $f$ is the external force, the governing equation for the generalised-alpha scheme of Jansen et al. [78] is given as,

$$
\dot{u}_{n+\alpha_{m}}-\vartheta u_{n+\alpha_{f}}=f_{n+\alpha_{f}},
$$

with,

$$
\begin{aligned}
u_{n+\alpha_{f}} & =\left[1-\alpha_{f}\right] u_{n}+\alpha_{f} u_{n+1}, \\
f_{n+\alpha_{f}} & =\left[1-\alpha_{f}\right] f_{n}+\alpha_{f} f_{n+1}, \\
\dot{u}_{n+\alpha_{m}} & =\left[1-\alpha_{m}\right] \dot{u}_{n}+\alpha_{m} \dot{u}_{n+1}, \\
u_{n+1} & =u_{n}+\Delta t\left[[1-\gamma] \dot{u}_{n}+\gamma \dot{u}_{n+1}\right], \\
\dot{u}_{n+1} & =\frac{1}{\gamma \Delta t}\left[u_{n+1}-u_{n}\right]+\frac{\gamma-1}{\gamma} \dot{u}_{n} .
\end{aligned}
$$

Here, $\Delta t$ is the time step size, and $\alpha_{m}, \alpha_{f}$ and $\gamma$ are the parameters of the time integration scheme. The generalised-alpha scheme is second-order accurate if the parameters are chosen such that

$$
\alpha_{m}=\frac{1}{2} \frac{3-\rho_{\infty}}{1+\rho_{\infty}} ; \quad \alpha_{f}=\frac{1}{1+\rho_{\infty}} ; \quad \gamma=\frac{1}{2}+\alpha_{m}-\alpha_{f}, \quad \text { for } 0 \leq \rho_{\infty} \leq 1,
$$

where, $\rho_{\infty}$ is the spectral radius at infinite time step size, and it controls the amount of numerical damping. For $\rho_{\infty}=0$, this scheme is spectrally equivalent to the second-order accurate backward differentiation formula (BDF2). The interested reader is referred to Jansen et al. [78] for the comprehensive details on the spectral characteristics of the generalised-alpha time integration scheme, and to Kadapa et al. [79] for its extension to structural dynamics problems.

\subsection{Monolithic scheme}

In a monolithic approach for FSI problems, the coupled equations (16) are all solved together for the solution variables in the solid and fluid sub-systems. Using the generalised-alpha scheme, the system of equations for the monolithic scheme for the solution of equations (16) can be written as,

$$
\begin{aligned}
\dot{d}_{n+\alpha_{m}^{s}}^{s}-v_{n+\alpha_{f}^{s}}^{s} & =0, \\
\alpha \dot{v}_{n+\alpha_{m}^{s}}^{s}+\omega^{2} d_{n+\alpha_{f}^{s}}^{s} & =f_{n+\alpha_{f}^{s}}^{s}, \\
{[1-\alpha] \dot{v}_{n+\alpha_{m}^{f}}^{f}+2 \xi \omega v_{n+\alpha_{f}^{f}}^{f} } & =f_{n+\alpha_{f}^{f}}^{f}, \\
v_{n+1}^{s} & =v_{n+1}^{f}, \\
f_{n+1}^{s}+f_{n+1}^{f} & =0 .
\end{aligned}
$$

With $v_{n}^{s}=v_{n}^{f}$ and $f_{n+1}^{s}=-f_{n+1}^{f}=f_{n+1}$, the system of equations 25 can be written in the matrix-vector form as, 


$$
\left\{\begin{array}{c}
d_{n+1}^{s} \\
v_{n+1}^{s} \Delta t \\
\dot{d}_{n+1}^{s} \Delta t \\
\dot{v}_{n+1}^{s} \Delta t^{2} \\
\dot{v}_{n+1}^{f} \Delta t^{2} \\
f_{n+1}^{f} \Delta t^{2}
\end{array}\right\}=\left[\mathbf{A}_{\text {mono }}\right]\left\{\begin{array}{c}
d_{n}^{s} \\
v_{n}^{s} \Delta t \\
\dot{d}_{n}^{s} \Delta t \\
\dot{v}_{n}^{s} \Delta t^{2} \\
\dot{v}_{n}^{f} \Delta t^{2} \\
f_{n} \Delta t^{2},
\end{array}\right\}
$$

where, $\mathbf{A}_{\text {mono }}$ is the amplification matrix for the monolithic scheme.

\subsection{Staggered scheme}

Using the generalised-alpha scheme, the governing equations for solution of equations (16) with the staggered approach are given as

$$
\begin{aligned}
f_{n+1}^{s^{P}} & =2 f_{n}^{s}-f_{n-1}^{s}, \\
f_{n+\alpha_{f}^{s}}^{s} & =\alpha_{f}^{s} f_{n+1}^{s^{P}}+\left[1-\alpha_{f}^{s}\right] f_{n}^{s}, \\
\dot{d}_{n+\alpha_{m}^{s}}^{s}-v_{n+\alpha_{f}^{s}}^{s} & =0, \\
\alpha \dot{v}_{n+\alpha_{m}^{s}}^{s}+\omega^{2} d_{n+\alpha_{f}^{s}}^{s} & =f_{n+\alpha_{f}^{s}}^{s}, \\
{[1-\alpha] \dot{v}_{n+\alpha_{m}^{f}}^{f}+2 \xi \omega v_{n+\alpha_{f}^{f}}^{f} } & =f_{n+\alpha_{f}^{f}}^{f}, \\
f_{n+\alpha_{f}^{f}}^{f} & =\alpha_{f}^{f} f_{n+1}^{f^{*}}+\left[1-\alpha_{f}^{f}\right] f_{n}^{f}, \\
f_{n+1}^{s} & =-\beta f_{n+1}^{f^{*}}+[1-\beta] f_{n+1}^{s^{P}}, \\
v_{n+1}^{s} & =v_{n+1}^{f}, \\
f_{n+1}^{s}+f_{n+1}^{f} & =0 .
\end{aligned}
$$

The above system of equations (27) can be written in the matrix-vector form as,

$$
\left\{\begin{array}{c}
d_{n+1}^{s} \\
\dot{d}_{n+1}^{s} \Delta t \\
v_{n+1}^{s} \Delta t \\
\dot{v}_{n+1}^{s} \Delta t^{2} \\
\dot{v}_{n+1}^{f} \Delta t^{2} \\
f_{n+1} \Delta t^{2} \\
f_{n} \Delta t^{2}
\end{array}\right\}=\left[\mathbf{A}_{\text {stag }}\right]\left\{\begin{array}{c}
d_{n}^{s} \\
\dot{d}_{n}^{s} \Delta t \\
v_{n}^{s} \Delta t \\
\dot{v}_{n}^{s} \Delta t^{2} \\
\dot{v}_{n}^{f} \Delta t^{2} \\
f_{n} \Delta t^{2} \\
f_{n-1} \Delta t^{2}
\end{array}\right\}
$$

where, $\mathbf{A}_{\text {stag }}$ is the amplification matrix for the staggered scheme. Using the field variables for the model problem, the pseudocode for the staggered scheme is shown in Algorithm. 1.

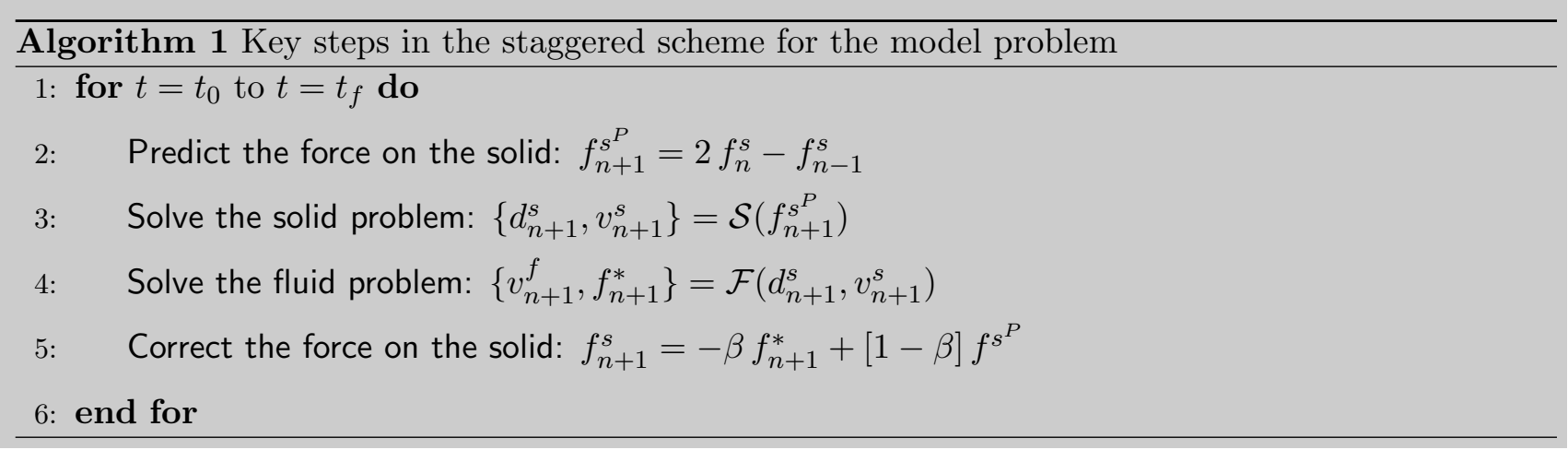


Remark 2: The staggered scheme has also been experimented with third-order $\left(f_{n+1}^{s^{P}}=3 f_{n}^{s}-3 f_{n-1}^{s}+f_{n-2}^{s}\right)$ and fourth-order $\left(f_{n+1}^{s^{P}}=4 f_{n}^{s}-6 f_{n-1}^{s}+4 f_{n-2}^{s}-f_{n-3}^{s}\right)$ force predictors. Although these higher-order force predictors improve the accuracy of the staggered scheme for problems with significant added-mass, i.e., smaller values of $m^{*}$ or $\alpha$, it has been observed that their stability limits are much more restrictive (with respect to $\alpha$ ) than the second-order predictor. Therefore, they are not pursued in this work.

Remark 3: Our experience indicates that the instability condition for the partitioned FSI schemes based on the displacement predictors (for the solid problem) is much more restrictive with respect to $m^{*}$ when compared with the one based on the force predictors used in the present work. Since there is no unique way of designing a staggered scheme using displacement predictors, the interested reader can compute the stability limits for the staggered scheme of their choice by using the linear model problem considered in this work.

Remark 4: For adaptive time stepping, the force predictor given in Eq. 27a is to be modified as

$$
f_{n+1}^{s^{P}}=[1+\Lambda] f_{n}^{s}-\Lambda f_{n-1}^{s}, \quad \text { with } \quad \Lambda=\frac{\Delta t_{n+1}}{\Delta t_{n}},
$$

where, $\Delta t_{n+1}$ and $\Delta t_{n}$ are the time step sizes, respectively, for the current and previously converged time steps.

\subsection{Stability and accuracy analysis}

Analytical proof of stability and accuracy of systems $(26)$ and $(28)$ is quite challenging. In this work, we assess the spectral characteristics of the monolithic and staggered schemes by evaluating the spectral radii of the corresponding amplification matrices for several combinations of system parameters. The spectral radius of a matrix $\mathbf{A}$ is defined as the largest value of its eigenvalues [82, 83]. A system is said to be stable for the chosen parameter values if the spectral radius of its amplification matrix is less than or equal to one; otherwise, the system is unstable.

Plots of spectral radii for four different values of $m^{*}, 10,2,0.2$ and 0.02 , are shown in Fig. 5 for $\xi=0$ and $\xi=0.1$. The numerical solutions computed with the staggered scheme for the same $m^{*}$ values are shown in Fig. 6. The important characteristics of the staggered scheme are:

(i) The staggered scheme is second-order accurate in time.

(ii) The leading error term for $\xi=0$ and $\rho_{\infty}^{s}=\rho_{\infty}^{f}=0$ is obtained as,

$$
\varepsilon\left(\mathcal{O}\left(\Delta t^{2}\right)\right)=\frac{4\left[3+2 \beta\left[1+m^{*}\right]\right]}{27 m^{*}} .
$$

Although the accuracy of the staggered scheme deteriorates as $m^{*}$ approaches zero, accurate numerical results can be obtained for the values of $m^{*}$ that are encountered in real-world applications, as demonstrated with the numerical examples of galloping of a square and vortex-induced vibrations of a circular cylinder, see Section 5 . Moreover, since the scheme is second-order accurate, the time step size required for obtaining accurate numerical results is not prohibitively small.

(iii) For the unconditional stability of the staggered scheme for the undamped case, i.e., $\xi=0$, the condition on the relaxation parameter $\beta$ is given by

$$
\beta \leq \frac{4}{3+\rho_{\infty}} \alpha, \quad \text { for } \quad \rho_{\infty}^{s}=\rho_{\infty}^{f}=\rho_{\infty} .
$$

This condition implies that smaller values of $\beta$ are required for smaller values of $\alpha$, which is the case for problems with significant added-mass. The condition for unconditional stability becomes very complicated for the case with damping. Therefore, the relaxation parameter $\beta$ is chosen based on numerical experiments.

(iv) The amount of numerical damping for the monolithic scheme remains the user-specified value, and is independent of the relaxation parameter $\beta$, as shown in Fig. 5. However, for the staggered scheme, the amount of numerical damping in the overall algorithm is a complicated function of $\rho_{\infty}^{s}, \rho_{\infty}^{f}$ and $\beta$. Using the limit case analysis as $\Delta t \rightarrow \infty$, the relation for the numerical damping is obtained as,

$$
\lim _{\Delta t \rightarrow \infty} \rho=\max \left(\rho_{\infty}^{s}, \rho_{\infty}^{f}, \sqrt{1-\beta}\right) .
$$


This means that, for small values of $\beta$, the spectral radius approaches one, since $\sqrt{1-\beta} \approx 1$ for $\beta \approx 0.0$. This is the case irrespective of the physical damping and the user-specified numerical damping, as shown in Fig. 5 . 


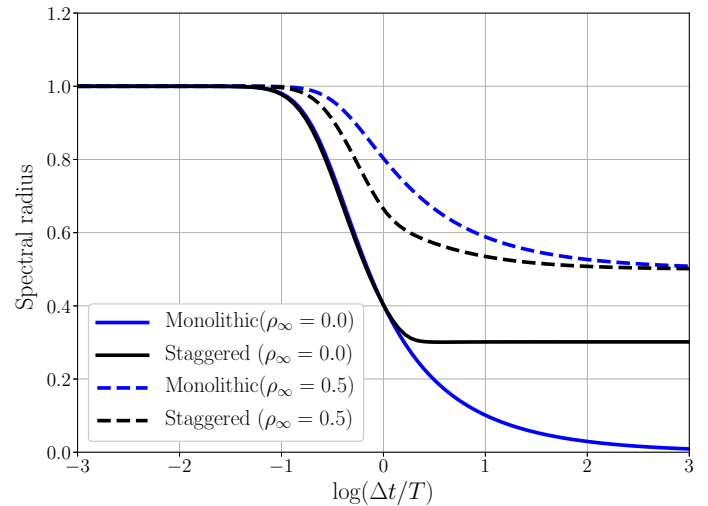

(a) $m^{*}=10.0, \xi=0.0$

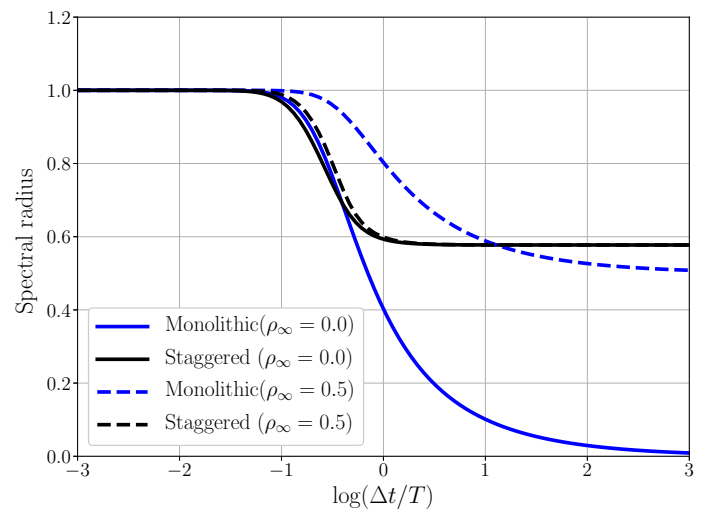

(c) $m^{*}=2.0, \xi=0.0$

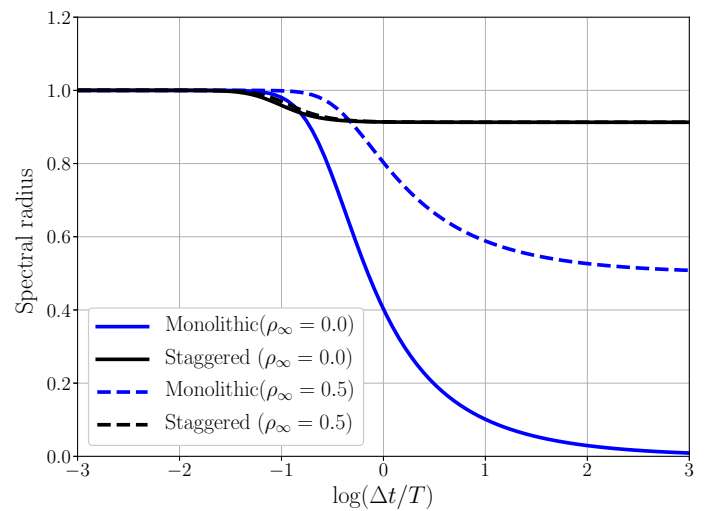

(e) $m^{*}=0.2, \xi=0.0$

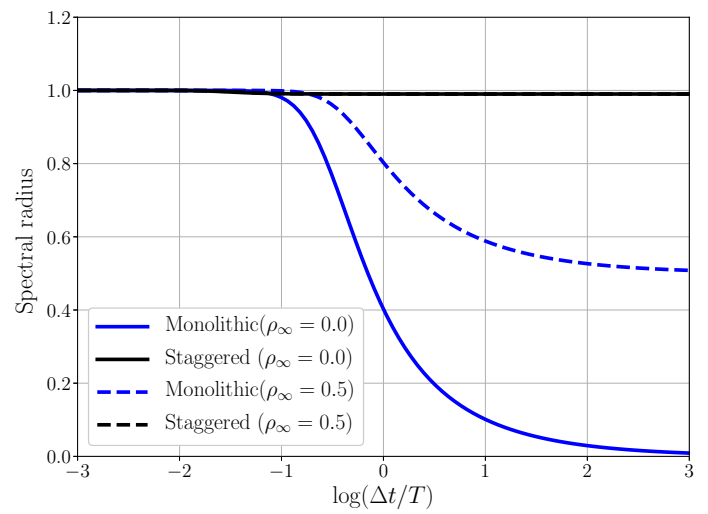

(g) $m^{*}=0.02, \xi=0.0$

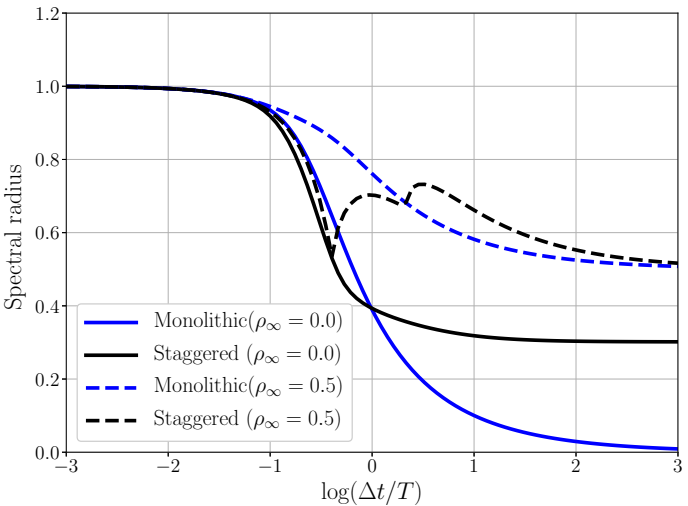

(b) $m^{*}=10.0, \xi=0.1$

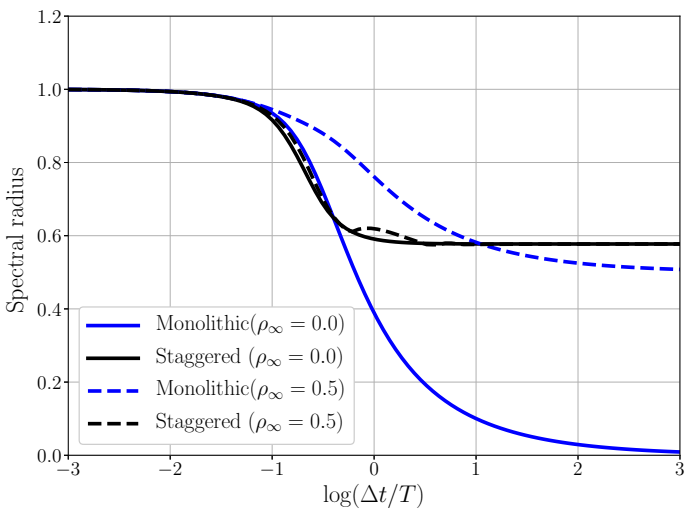

(d) $m^{*}=2.0, \xi=0.1$

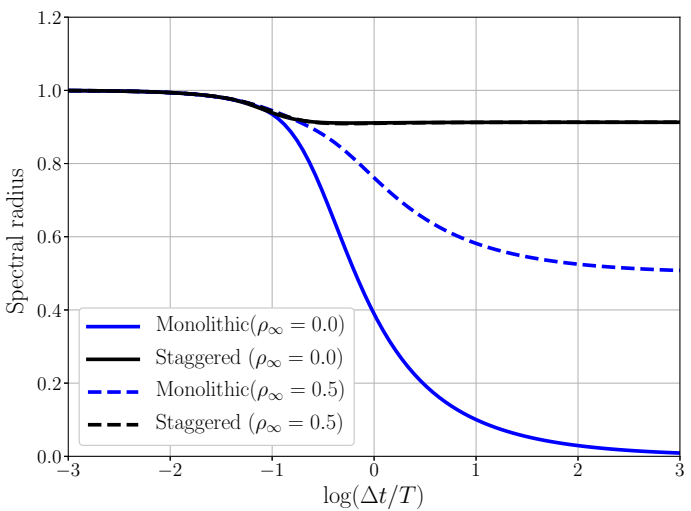

(f) $m^{*}=0.2, \xi=0.1$

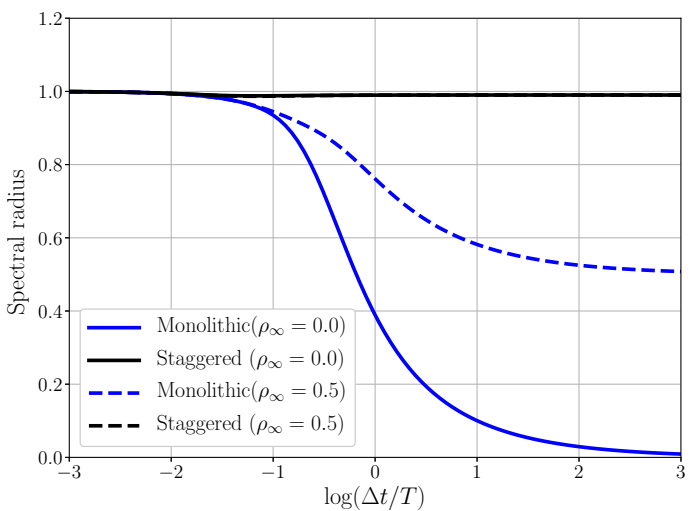

(h) $m^{*}=0.02, \xi=0.1$

Figure 5: Model problem: spectral radii for $\omega=1.0, m_{f}=1.0, \rho_{\infty}^{s}=\rho_{\infty}^{f}=\rho_{\infty}$ and $\beta=\alpha$. 


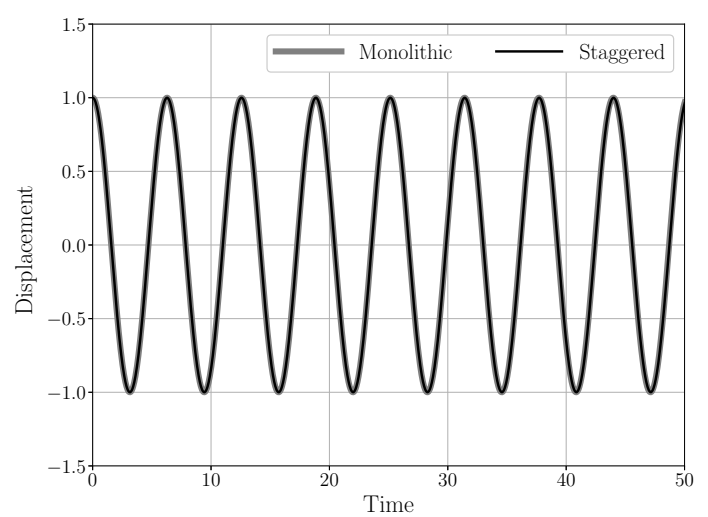

(a) $m^{*}=10.0, \xi=0.0$

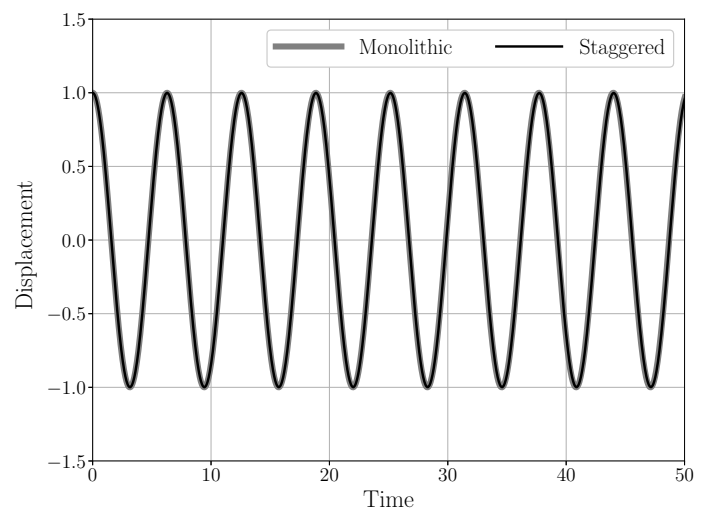

(c) $m^{*}=2.0, \xi=0.0$

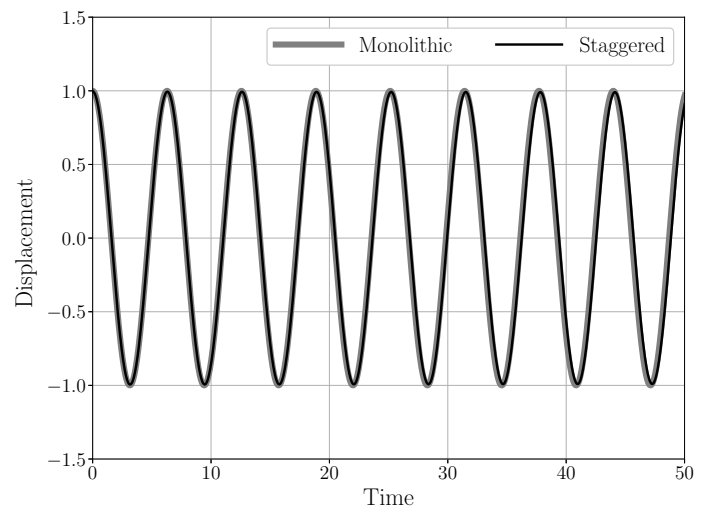

(e) $m^{*}=0.2, \xi=0.0$

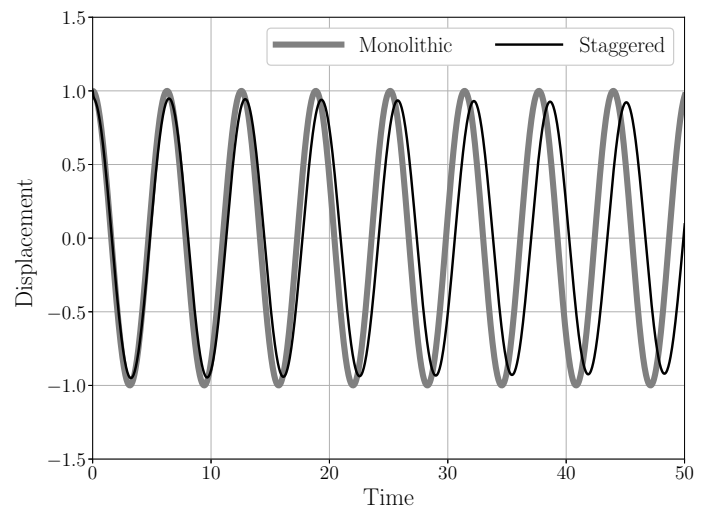

(g) $m^{*}=0.02, \xi=0.0$

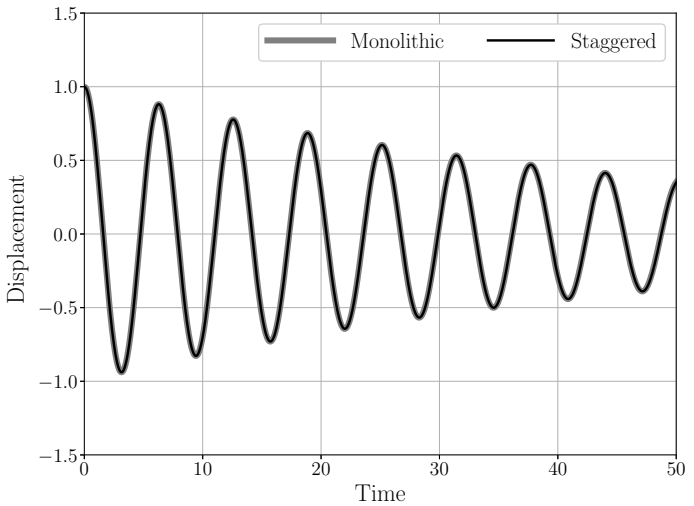

(b) $m^{*}=10.0, \xi=0.02$

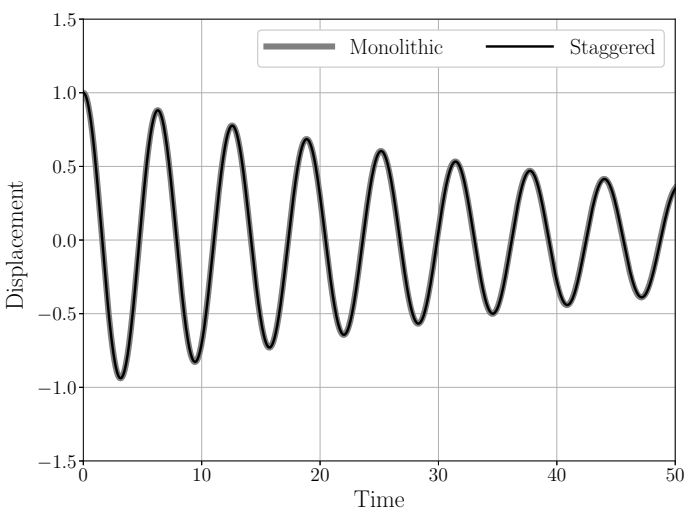

(d) $m^{*}=2.0, \xi=0.02$

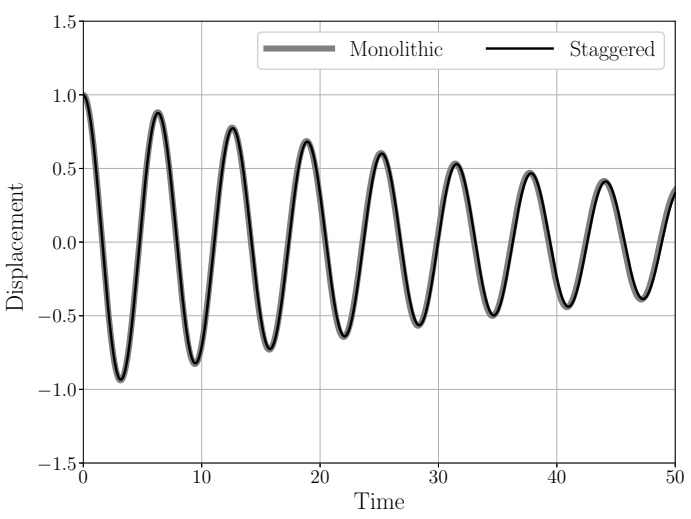

(f) $m^{*}=0.2, \xi=0.02$

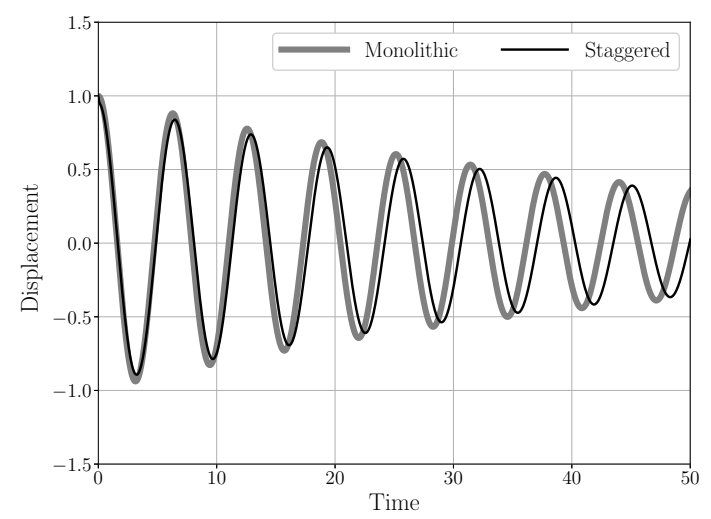

(h) $m^{*}=0.02, \xi=0.02$

Figure 6: Model problem: spectral radii for $\omega=1.0, m_{f}=1.0, \rho_{\infty}^{s}=\rho_{\infty}^{f}=0.0, \beta=\alpha$ and $\Delta t=T / 200$. 


\section{Numerical examples}

The suitability of the staggered scheme for applications in FSI problems, especially those involving lightweight rigid solids often encountered in the interaction of floating bodies in Ocean Engineering and marine structures [84-87], is demonstrated using the examples of galloping of a square, vortex-induced vibrations of a circular cylinder and an extremely lightweight rectangular block rising in a fluid chamber. In this work, the flow is assumed to be isothermal, laminar, viscous and incompressible.

The proposed staggered scheme for resolving fluid-solid interactions is based on the Dirichlet-Neumann coupling; therefore, the input to the fluid solver is the position and velocity of the solid body and the input to the solid solver is the force exerted by the fluid. Thus, the proposed scheme is non-intrusive, meaning that it can be used for combining different fluid and solid solvers by treating them as black-box solvers. To demonstrate this non-intrusive nature of the staggered scheme, two different fluid solvers are considered in this work. The first fluid solver is based on the fictitious-domain/distributed Lagrangian multiplier methodology proposed in Kadapa et al. [27] and the second one is based on the cut-cell based approach proposed in Kadapa et al. [14, 15, 104]. Both fluid solvers are based on the concept of unfitted meshes. The reader is referred to [11, 14, 15, 27, 104] for the comprehensive details of the formulation for the fluid solvers.

In all the numerical simulations, the spectral radius is considered as $\rho_{\infty}^{s}=\rho_{\infty}^{f}=0$. The guidelines for the selection of the relaxation parameter $\beta$ are outlined in Section 6 .

\subsection{Galloping of a square}

Galloping is a self-excited instability which results in high-amplitude and low-frequency oscillations unlike lock-in which is caused by resonance [88, 89]. The geometry and boundary conditions of the problem are as shown in Fig. 7(a), The length of the side of the square is $D=1$. The properties of the fluid are: density, $\rho^{f}=1$, and dynamic viscosity, $\mu^{f}=0.01$. The value of $v_{\infty}$ at the inlet is adjusted to match the Reynolds number $(R e)$ of interest, where, $R e=\rho^{f} D v_{\infty} / \mu^{f}$.

The simulation framework used for this example is the fictitious-domain/distributed Lagrange multiplier methodology [27]. The main reason behind using this framework is its ability to compute numerical solutions of FSI problems using a monolithic scheme which serve as the reference solutions for assessing the accuracy of the results obtained with the proposed staggered scheme. Before presenting the results for galloping, spatial and temporal convergence of the formulation are assessed by simulating the unsteady flow past a fixed square.

\subsubsection{Spatial and temporal convergence study - flow past a fixed square}

For the convergence study, unsteady flow past a fixed square for $R e=100$ and $R e=200$ is considered. The finite element mesh with multiple levels of hierarchical refinement, as shown in Fig. 7, is used for the analysis. The number of points per side of the square is 10 and 20, respectively, for the level-2 (L2) and level-3 (L3) meshes. The number of DOFs, including Lagrange multipliers, for the fluid problem is 32441 and 46867 , respectively, for the level-2 and level-3 meshes. Two different time step sizes, $\Delta t=0.1$ and $\Delta t=0.05$ are considered for each mesh. The graphs of the evolution of the lift coefficient, as shown in Figs. 8 and 9 , respectively, for $R e=100$ and $R e=200$, indicate convergence of the numerical solutions with respect to spatial and temporal discretisation. The computed values of mean drag coefficient $\left(C_{D_{\text {mean }}}\right)$, RMS lift coefficient $\left(C_{L_{\mathrm{rms}}}\right)$ and Strouhal number $(S t)$ are tabulated in Tables. 1 and 2, respectively, for $R e=100$ and $R e=200$. As shown, the present numerical results match well with the corresponding values from the published literature [90-98]. 


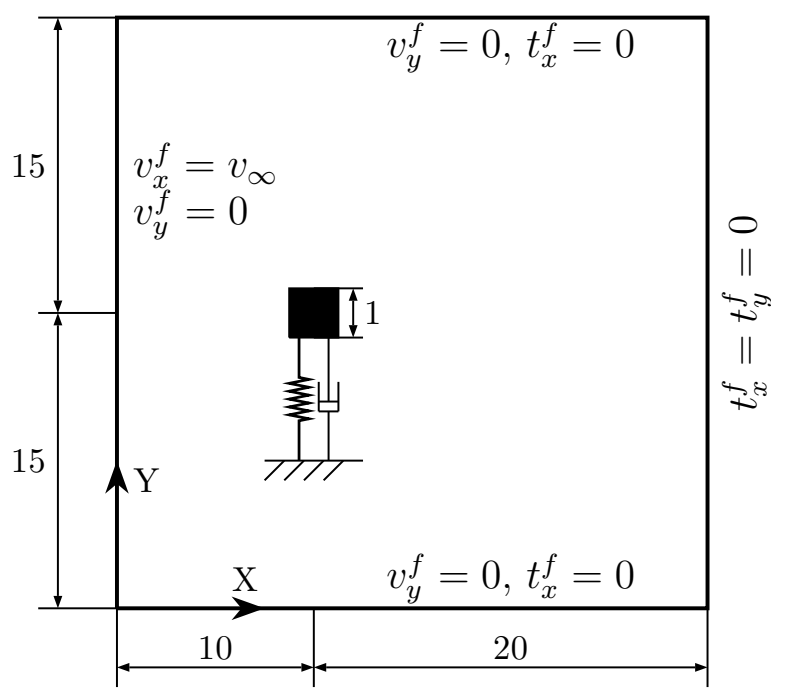

(a)

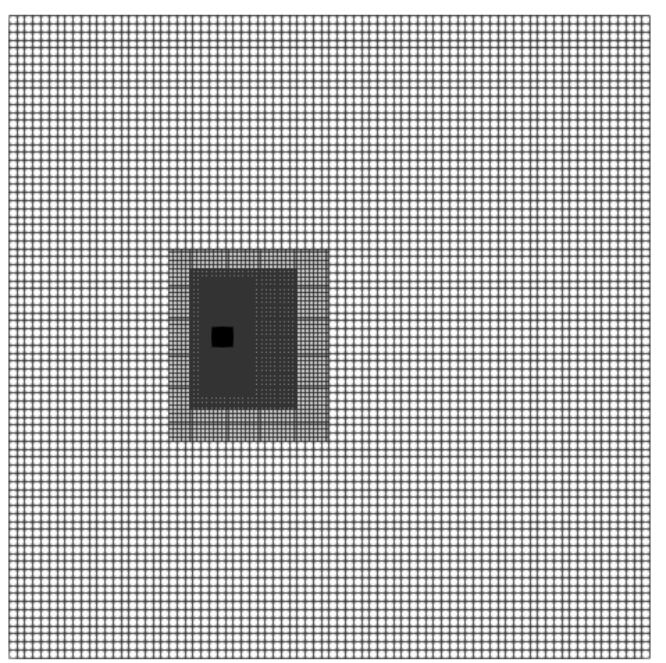

(b)

Figure 7: Flow past a square: (a) geometry and boundary conditions and (b) the finite element mesh used for the simulation.

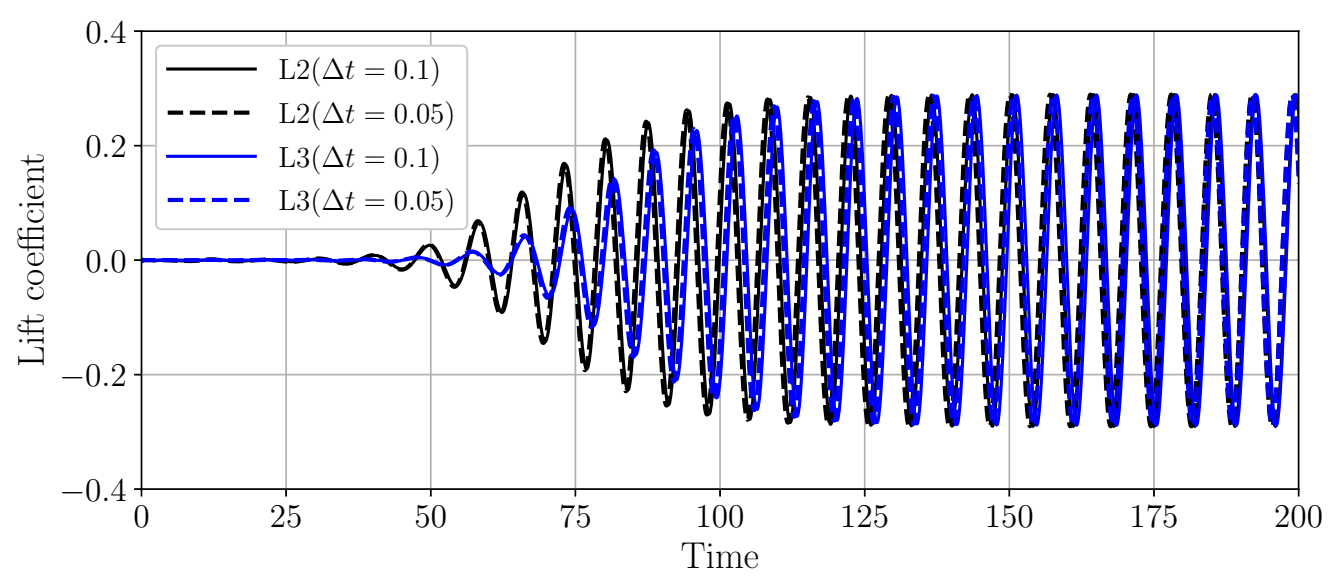

Figure 8: Flow past a fixed square: evolution of the lift coefficient $\left(C_{L}\right)$ for $R e=100$.

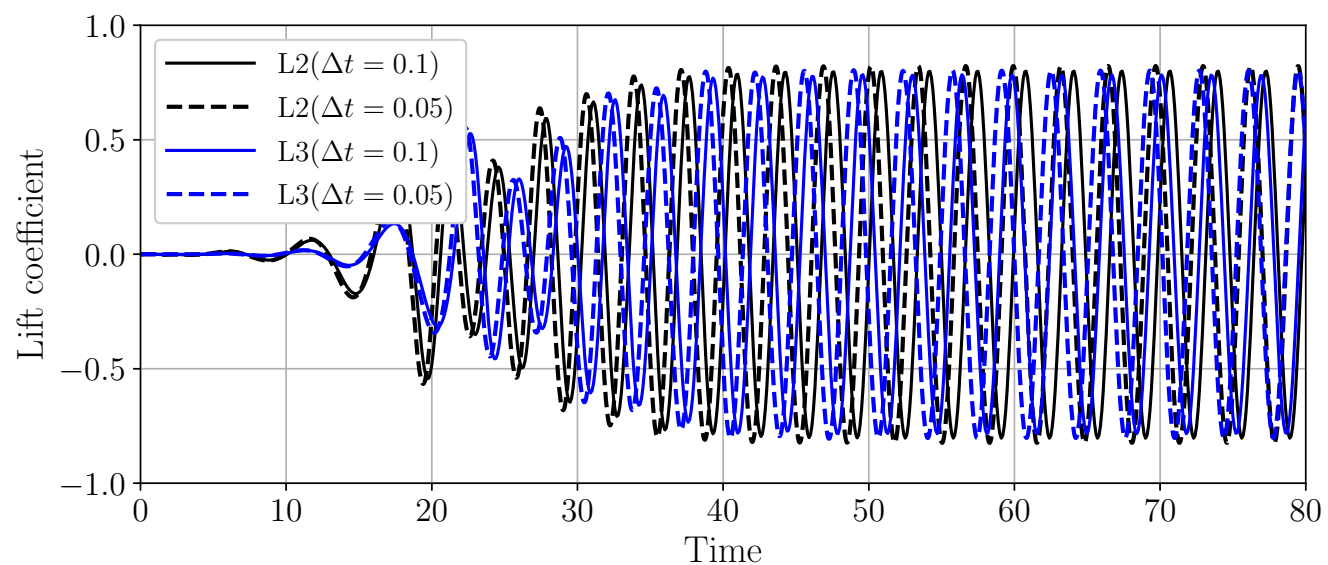

Figure 9: Flow past a fixed square: evolution of the lift coefficient $\left(C_{L}\right)$ for $R e=200$. 


\begin{tabular}{|l|c|c|c|}
\hline Study & $C_{D_{\text {mean }}}$ & $C_{L_{\mathrm{rms}}}$ & $S t$ \\
\hline Sharma and Eswaran [90] & 1.4936 & 0.1922 & 0.1488 \\
Sen et al. [91] & 1.5287 & 0.1928 & 0.1452 \\
Sahu et al. 92] & 1.4878 & 0.1880 & 0.1486 \\
Sohankar et al. [93] & 1.4770 & 0.1560 & 0.1460 \\
Singh et al. [94] & 1.5100 & 0.1600 & 0.1470 \\
Darekar and Sherwin (3D) [95] & 1.4860 & 0.1860 & 0.1460 \\
Zhao et al. [96] & 1.4520 & 0.1908 & 0.1447 \\
\hline Present (Level-2, $\Delta t=0.1)$ & 1.6136 & 0.1876 & 0.1440 \\
Present (Level-2, $\Delta t=0.05)$ & 1.6136 & 0.1877 & 0.1420 \\
Present (Level-3, $\Delta t=0.1)$ & 1.5523 & 0.1843 & 0.1460 \\
Present (Level-3, $\Delta t=0.05)$ & 1.5525 & 0.1842 & 0.1460 \\
\hline
\end{tabular}

Table 1: Flow past a fixed square: mean drag coefficient $\left(C_{D_{\text {mean }}}\right)$, RMS lift coefficient $\left(C_{L_{\mathrm{rms}}}\right)$ and Strouhal number (St) for $R e=100$.

\begin{tabular}{|l|c|c|c|}
\hline Study & $C_{D_{\text {mean }}}$ & $C_{L_{\mathrm{rms}}}$ & $S t$ \\
\hline Sohankar et al. [97] & 1.4600 & 0.3200 & 0.1700 \\
Singh et al. (3D) [94] & 1.5400 & 0.5500 & 0.1420 \\
Joly et al. [98] & 1.4400 & 0.4200 & 0.1510 \\
\hline Present (Level-2, $\Delta t=0.1)$ & 1.7830 & 0.5519 & 0.1509 \\
Present (Level-2, $\Delta t=0.05)$ & 1.7934 & 0.5658 & 0.1530 \\
Present (Level-3, $\Delta t=0.1)$ & 1.6320 & 0.5376 & 0.1470 \\
Present (Level-3, $\Delta t=0.05)$ & 1.6385 & 0.5501 & 0.1470 \\
\hline
\end{tabular}

Table 2: Flow past a fixed square: mean drag coefficient $\left(C_{D_{\text {mean }}}\right)$, RMS lift coefficient $\left(C_{L_{\mathrm{rms}}}\right)$ and Strouhal number (St) for $R e=200$.

\subsubsection{Galloping with mass ratio $m^{*}=20$}

First, we consider the simulation case for $m^{*}=20$ that has been originally proposed in Robertson et al. [99] and later studied in [7, 14, 28, 58, 100, 101]. For this case, the simulation parameters are: $m^{*}=20, R e=250$, reduced velocity, $U_{r}=\frac{v_{\infty}}{f_{n} D}=40$, and $\xi=0.0037$. The natural frequency for this system is, $f_{n}=0.0625 \mathrm{~Hz}$. Level-3 mesh with $\Delta t=0.05$ is used for performing simulations with both monolithic and staggered schemes. The relaxation parameter for the staggered scheme is $\beta=0.9(\approx \alpha)$. The displacement response of the square body obtained with the staggered scheme, as shown in Fig. 10, is in excellent agreement with that of the monolithic scheme. The values of displacement amplitude $(Y / D)$, frequency of rigid body oscillations $\left(f_{o}\right)$, and the frequency of vortex-shedding $\left(f_{v}\right)$ match well with the reference values, as shown in Table. 3. Thus, the numerical results obtained with this case establish the accuracy of the staggered scheme against a benchmark example. 


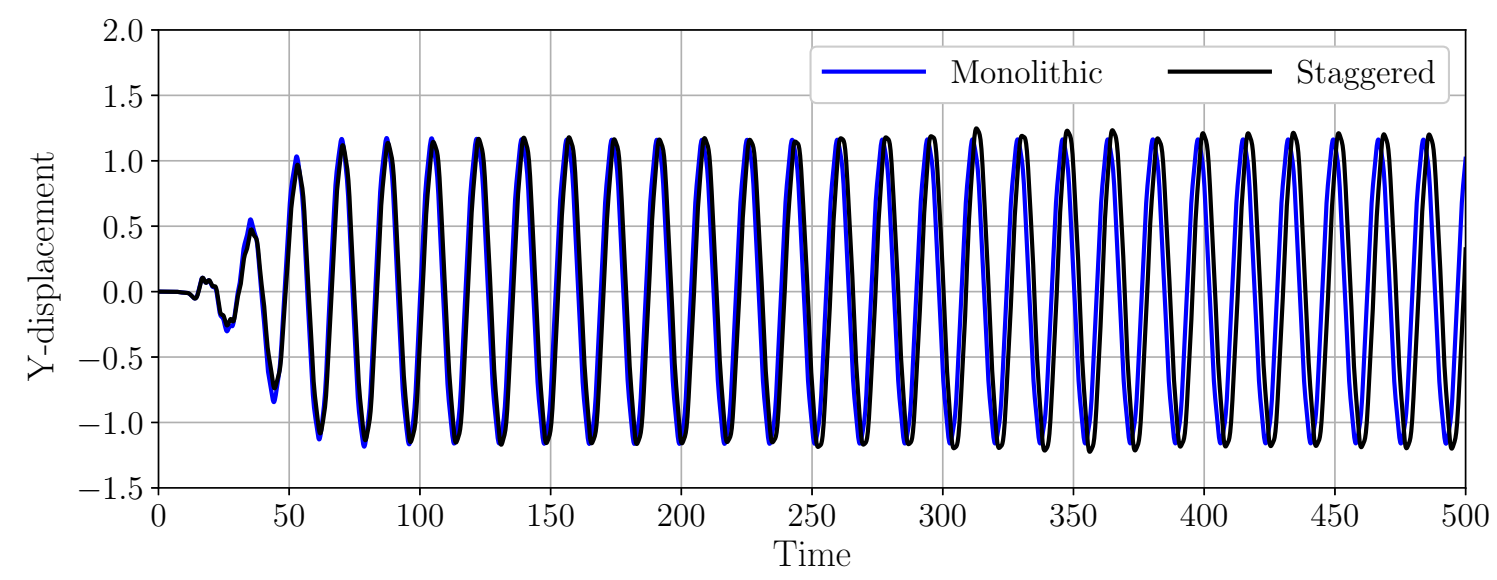

Figure 10: Galloping of a square: displacement response for $m^{*}=20$. For this case, $\beta=0.9$.

\begin{tabular}{|l|c|c|c|}
\hline Study & $Y / D$ & $f_{o} / f_{n}$ & $f_{v} / f_{n}$ \\
\hline Robertson et al. [99] & 1.150 & 0.938 & - \\
Dettmer and Perić [28] & 1.117 & 0.943 & 6.33 \\
He et al. [56] & 1.279 & 0.952 & 6.367 \\
He et al. [57] & 1.159 & 0.939 & 6.525 \\
Kadapa et al. [14] & 1.197 & 0.944 & - \\
Present - Monolithic & 1.174 & 0.939 & 6.488 \\
Present - Staggered & 1.180 & 0.958 & 6.489 \\
\hline
\end{tabular}

Table 3: Galloping of a square: displacement amplitude and frequency of oscillations for $m^{*}=20, R e=250, U r=40$ and $\xi=0.0037$.

\subsubsection{Galloping with mass ratio $0.01 \leq m^{*} \leq 5$}

Simulations are performed for different values of mass ratio in the range $0.01 \leq m^{*} \leq 5$ by keeping the rest of the parameters constant. The displacement response of the square for $m^{*}=\{5,2,0.2,0.02\}$, are presented in Fig. 11. These graphs illustrate that the displacement responses obtained with the proposed scheme are in excellent agreement with the respective solutions obtained with the monolithic scheme. The time step size of $\Delta t=0.02$ used for $m^{*}=\{2,0.2,0.02\}$ corresponds to only 100 time steps per one period of sustained oscillation. Successful simulations for even lower values of $m^{*}$ can also be performed by reducing the relaxation parameter and time step size appropriately, as demonstrated in Fig. 12 for $m^{*}=0.01$, and also in the following examples.

The presented results illustrate the exceptional accuracy of numerical results that can be obtained with the proposed scheme. It is remarkable that such accurate numerical results can be obtained with the proposed staggered scheme, especially considering that the time step size is large and that the scheme does not require any sub-iterations at each time step. The ability of the staggered scheme to compute accurate results using large time steps and without sub-iterations reaps significant computational benefits in performing large-scale 3D FSI simulations.

The graphs in Fig. 11 show that the amplitude of periodic oscillations of the square in galloping reduces substantially for low values of $m^{*}$. This behaviour of reduced amplitude of oscillations of a galloping body for low values of $m^{*}$ is consistent with the earlier observations reported in Joly et al. [98]. 


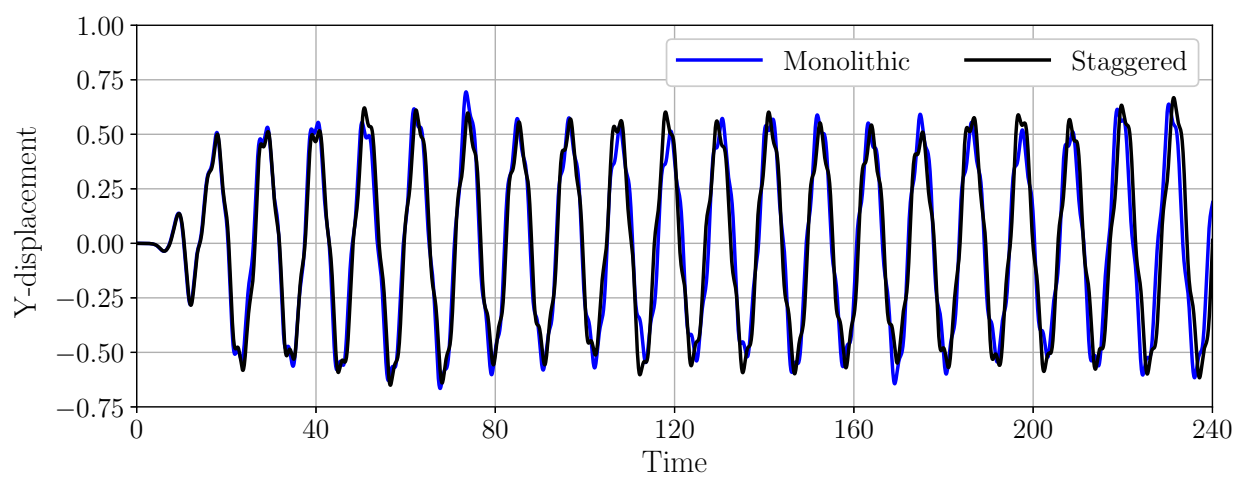

(a) $m^{*}=5(\beta=0.8, \Delta t=0.05)$

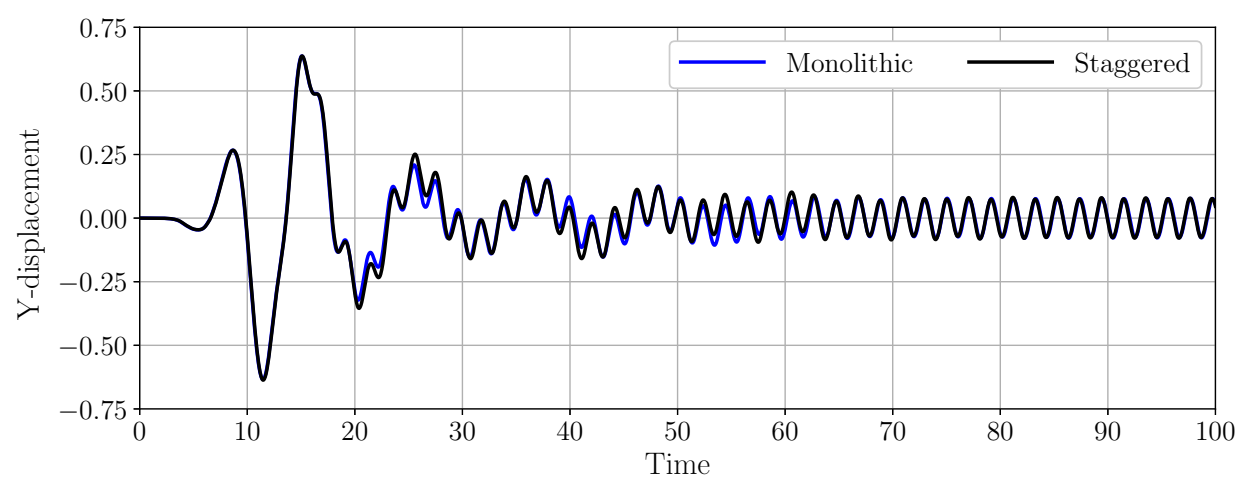

(b) $m^{*}=2(\beta=0.5, \Delta t=0.02)$

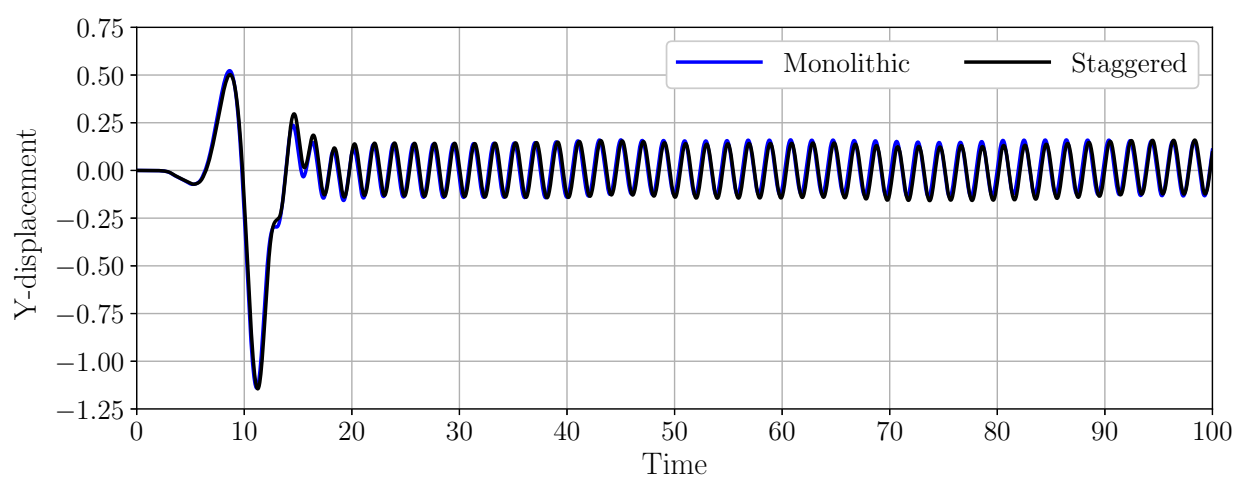

(c) $m^{*}=0.2(\beta=0.1, \Delta t=0.02)$

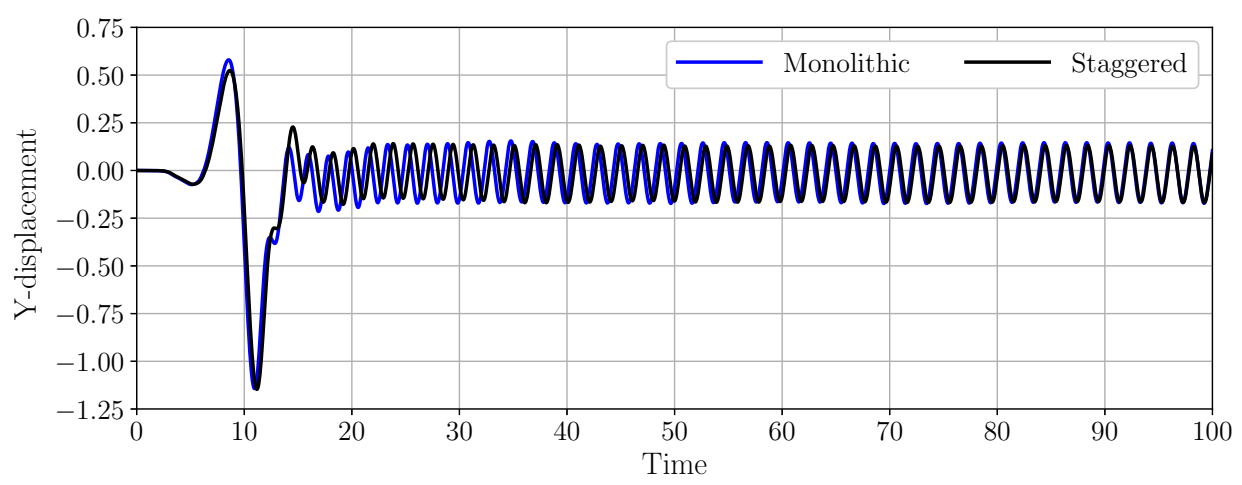

(d) $m^{*}=0.02(\beta=0.01, \Delta t=0.02)$

Figure 11: Galloping of a square: displacement response for four different values of $m^{*}$. The corresponding values of the relaxation parameter $\beta$ and time step size $\Delta t$ are indicated in the captions of subfigures. 


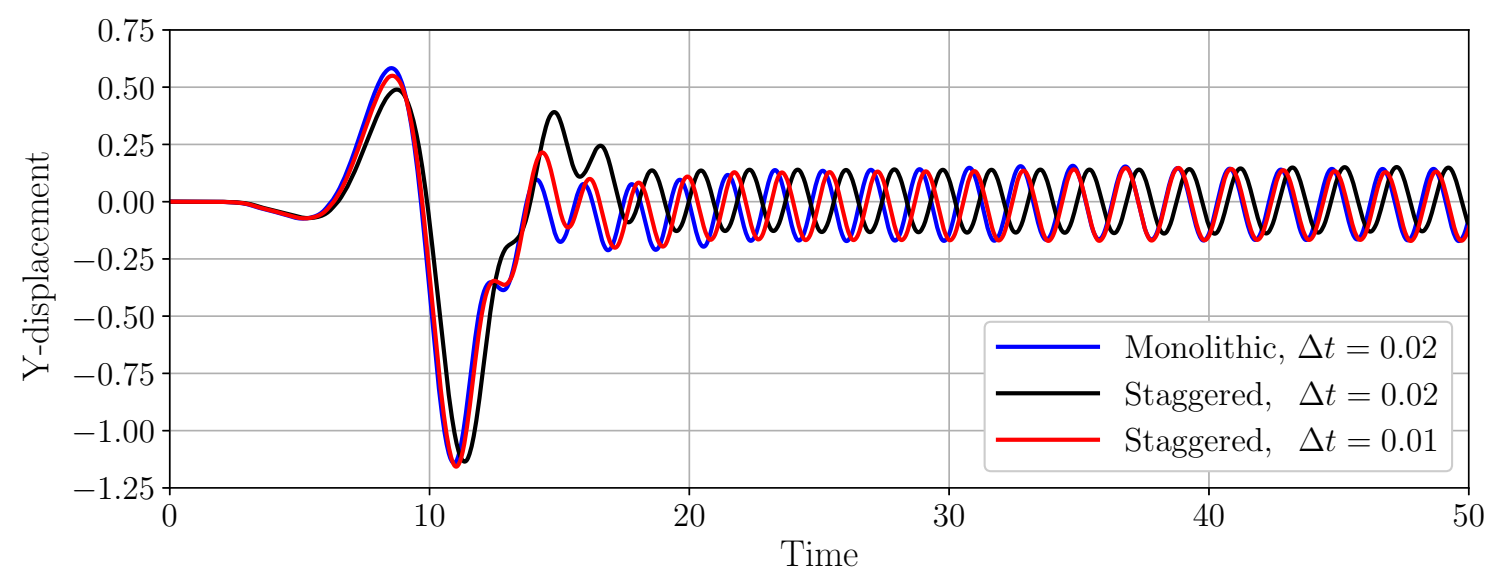

Figure 12: Galloping of a square: displacement response for $m^{*}=0.01$. Relaxation parameter, $\beta=0.005$.

\subsection{Vortex-induced vibrations of a circular cylinder}

This example is concerned with the problem of vortex-induced oscillations of a circular cylinder at $R e=100$. To demonstrate the non-intrusive nature of the staggered scheme, a different fluid solver from the one used for the previous example is considered. The fluid problem in this example is solved using the stabilised finite element formulation proposed in Kadapa et al. [11, 14, 15, 104]. The key difference between the formulation for the present example and previous example is in the technique used for imposing the kinematic constraint between the fluid and solid interface. While interface condition is enforced using distributed Lagrange multipliers in the fictitious domain method used in the previous example, it is enforced using the Nitsche method in the cut-cell-based method used in this example. For comprehensive details regarding the finite element formulations, we refer the reader to [11, 14, 15, 104]. The spatial and temporal convergences of the formulation for the example of flow past a fixed circular cylinder and the benchmark examples of transverse galloping of a square and rotational galloping a rectangle are already established in [14]. Therefore, convergence studies are not repeated in this work.

The geometry and boundary conditions of the fluid domain are shown in Fig. 13(a). Important parameters of the problem are: cylinder diameter, $D=1$; fluid density, $\rho^{f}=1$; fluid viscosity, $\mu^{f}=0.01$; inlet velocity, $v_{\infty}=1$; natural frequency, $f_{n}=0.2 \mathrm{~Hz}$; and damping ratio, $\xi=0$. For these parameters, the reduced velocity is, $U_{r}=\frac{v_{\infty}}{f_{n} D}=5$. The displaced mass of the fluid, $m^{f}$, is kept constant, and the solid mass and spring stiffness are adjusted based on the mass ratio, $m^{*}$. To accommodate the significant streamwise movement of the cylinder for low mass ratio values, the finite element mesh with extended refinement in the streamwise direction, as shown in Fig. 13(b), is used. The total number of DOFs for the fluid problem is approximately 125600 at each time step. The circumference of the cylinder is discretised with 80 straight edges. Simulations are performed for different values of $m^{*}$ in the range $0.01 \leq m^{*} \leq 10$. The time step for all the simulations is $\Delta t=0.05$, which corresponds to only 100 time steps per one natural time period of the spring-mass system. 


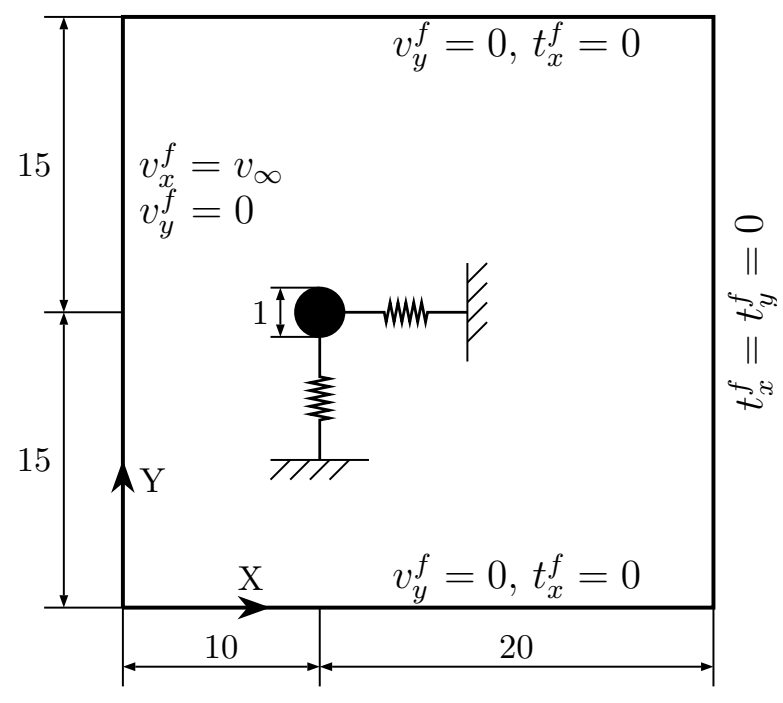

(a)

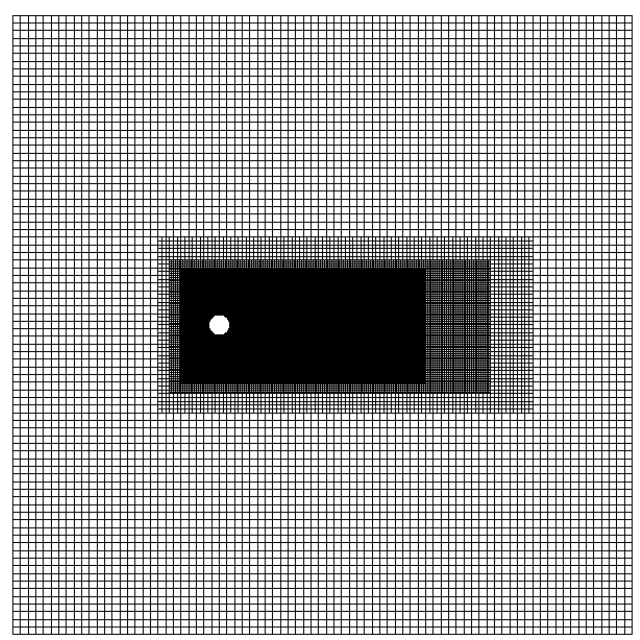

(b)

Figure 13: VIV of a circular cylinder: (a) geometry and boundary conditions and (b) the finite element mesh with three levels of hierarchical refinement.

\subsubsection{2-DOF motion with mass ratio $m^{*}=10$}

The relaxation parameter considered for this case is $\beta=0.45(\approx 0.5 \alpha)$. The evolution of force coefficients and displacements of the circular cylinder obtained with the staggered scheme is shown in Fig. 14. The amplitudes of Y-displacement and lift coefficient obtained in the present work are in good agreement with the results presented in Jaiman et al. [68], see Table 4 .
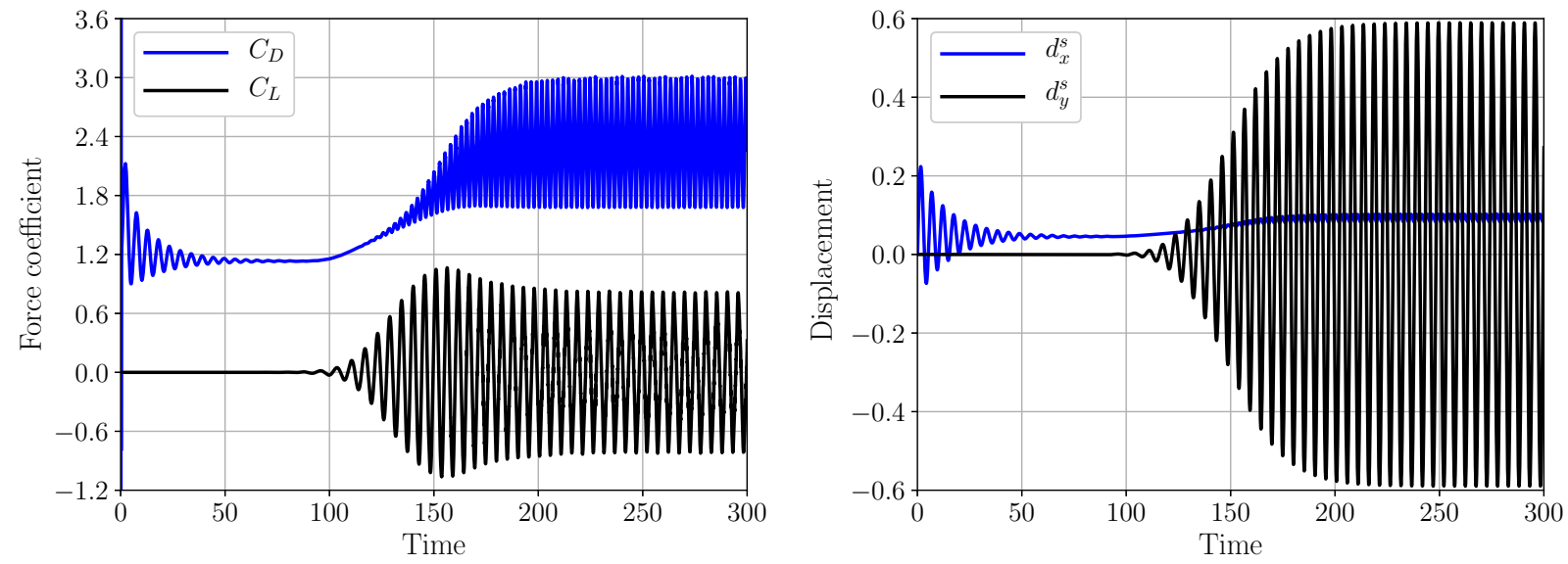

Figure 14: VIV of a circular cylinder: (a) force coefficients and (b) displacement response, for $m^{*}=10$. The relaxation parameter is $\beta=0.45$.

\begin{tabular}{|l|c|c|}
\hline Study & Displacement amplitude $(Y / D)$ & Lift coefficient $\left(C_{L}\right)$ \\
\hline Jaiman et al. [68] & 0.56 & 0.88 \\
Present & 0.59 & 0.83 \\
\hline
\end{tabular}

Table 4: VIV of a circular cylinder: displacement amplitude $(Y / D)$ and lift coefficient $\left(C_{L}\right)$ for $m^{*}=10$.

\subsubsection{2-DOF motion with mass ratio $0.1 \leq m^{*} \leq 2$}

Analogous to the studies performed in Jaiman et al. [68] and He [69], a number of simulations are performed with different mass ratio values in the range $0.1 \leq m^{*} \leq 2$ for the vortex-induced vibrations of a circular cylinder with 2-DOF motion. Note that the lowest $m^{*}$ value reported in Jaiman et al. [68] is 0.26, and in He 
[69] it is 0.298, while the lowest value in the present work for the circular cylinder with 2-DOF motion is 0.1. Simulations with even lower values for $m^{*}$ are performed using the 1-DOF model in the next subsection. The time step is $\Delta t=0.01$ for $t \leq 5$ and $\Delta t=0.05$ for the rest of the simulation.

The Lissajous orbits of the centre of mass of the cylinder for different values of $m^{*}$, as shown in Fig. 15 , demonstrate the sustained periodic oscillations resulting in the well-known 8-profile for the vortex-induced vibrations of a circular cylinder at low Reynolds numbers [68, 69, 76, 102, 103]. The evolution of force coefficients and displacements of the cylinder for $m^{*}=0.408$, as presented in Fig. 16, agree well with the results presented in He [69]. The contour plots of pressure at two different time instants are shown in Fig. 17. To the best of the author's knowledge, this is the first time in the literature vortex-induced vibrations of a circular with 2-DOF motion are reported for such a low value of 0.1 for the mass ratio.

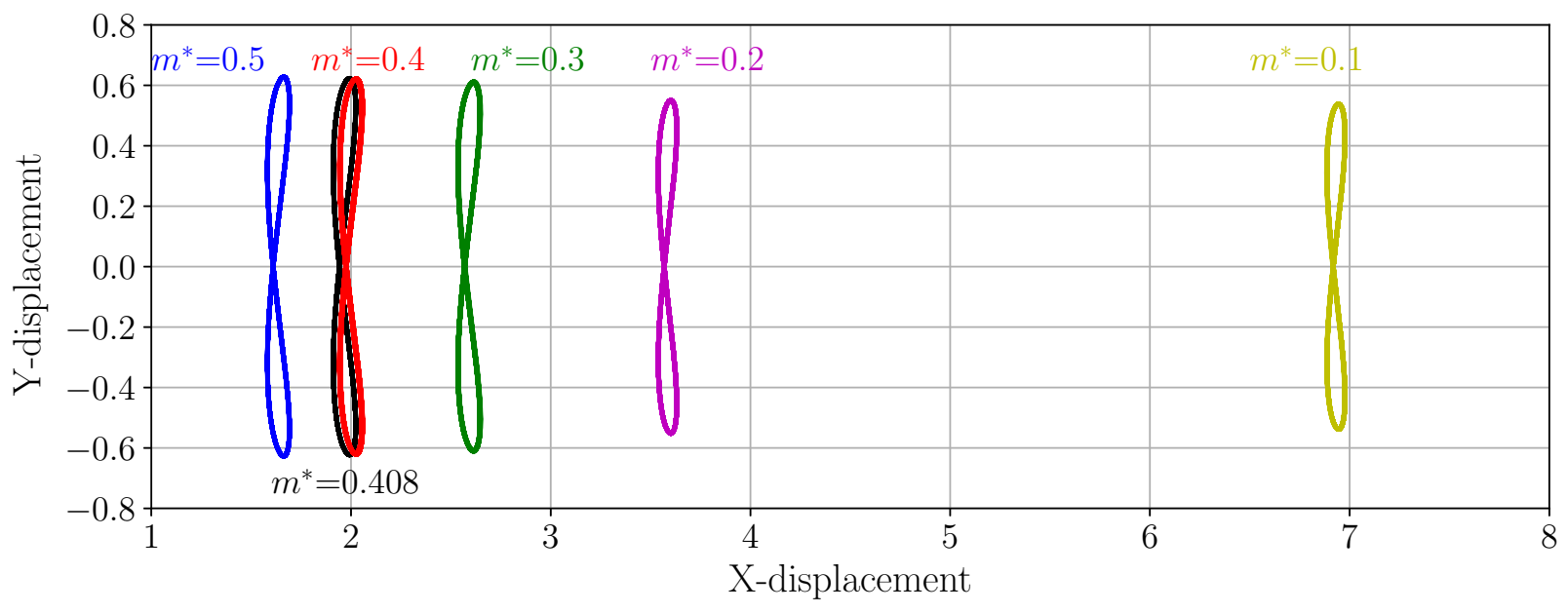

Figure 15: VIV of a circular cylinder: trajectories of the centre of mass of the cylinder for different $m^{*}$ values.
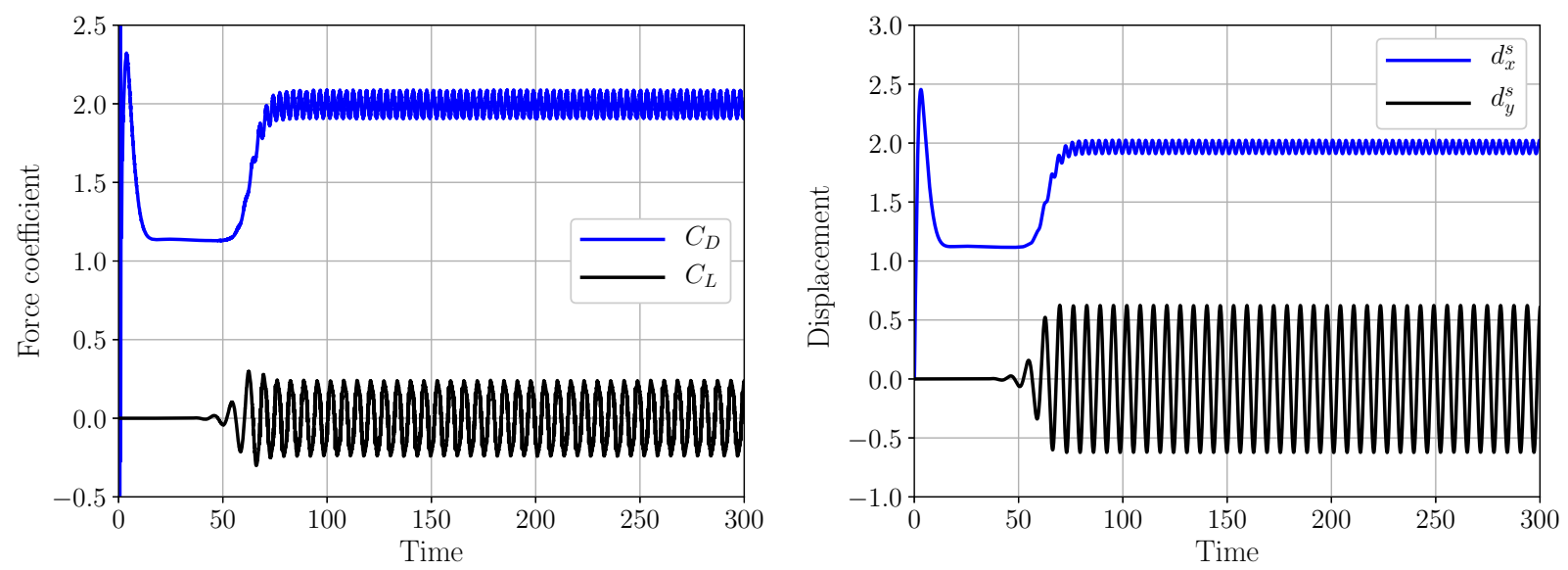

Figure 16: VIV of a circular cylinder: (a) force coefficients and (b) displacement response, for $m^{*}=0.408$. The relaxation parameter is $\beta=0.14$. 

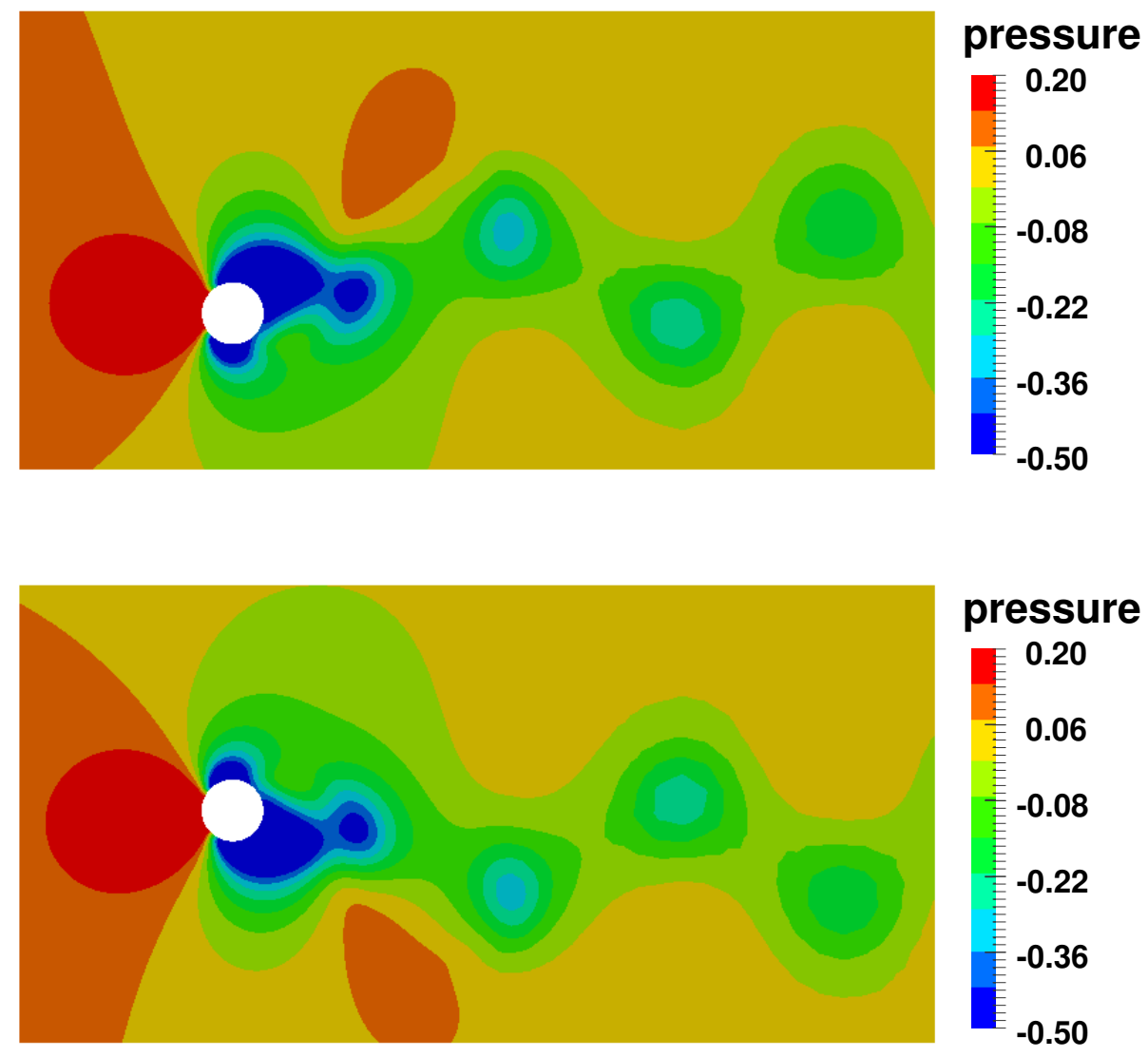

Figure 17: VIV of a circular cylinder: instantaneous contour plots of pressure at two different time instants for $m^{*}=0.408$.

\subsubsection{1-DOF motion with mass ratio $0.01 \leq m^{*}<0.1$}

The streamwise displacement of the cylinder becomes excessive for $m^{*}<0.1$, which requires an extension of the fluid domain further downstream. To avoid the associated computational cost, cylinder with only transverse DOF is considered for $0.01 \leq m^{*}<0.1$. Simulations are performed for different values of $m^{*}$ in the range $0.01 \leq m^{*}<0.1$ using a uniform time step of $\Delta t=0.05$ and the responses of the cylinder for four different values, $m^{*}=\{0.04,0.03,0.02,0.01\}$, are presented in Fig. 18 . The respective relaxation parameters used for these simulations are $0.02,0.015,0.01$ and 0.005 , which correspond to $\beta \approx 0.5 \alpha$. As shown in Fig. 18 , the response of the cylinder is smooth, and there is no significant difference in the amplitude and frequency of oscillations. The ever so slight increase in the time period of oscillations with decreasing $m^{*}$ is consistent with the expected behaviour: as the mass ratio decreases the added-mass increases, thereby increasing the effective mass. This increased effective mass reflects in the increased time period (or reduced frequency) of oscillations.

The results presented for $0.01 \leq m^{*}<0.1$ illustrate the remarkable ability of the proposed iteration-free staggered scheme in performing successful simulations of FSI problems involving extremely lightweight rigid solids. 


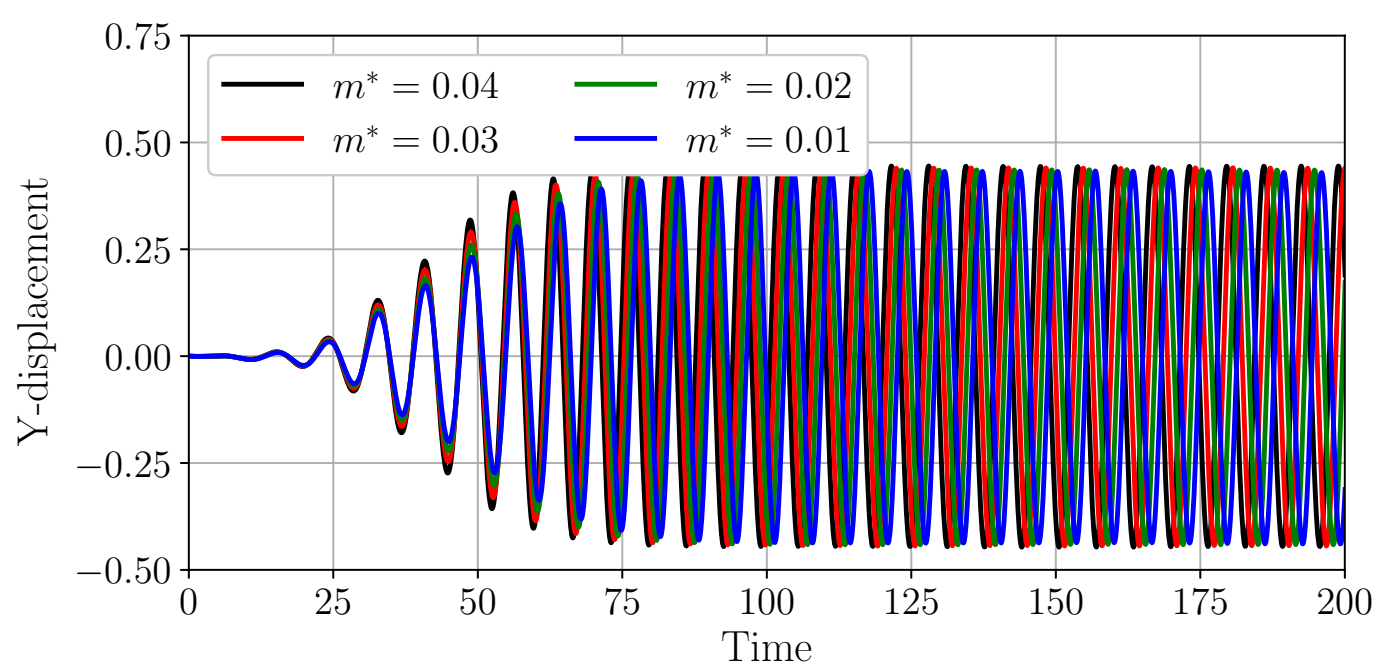

(a)

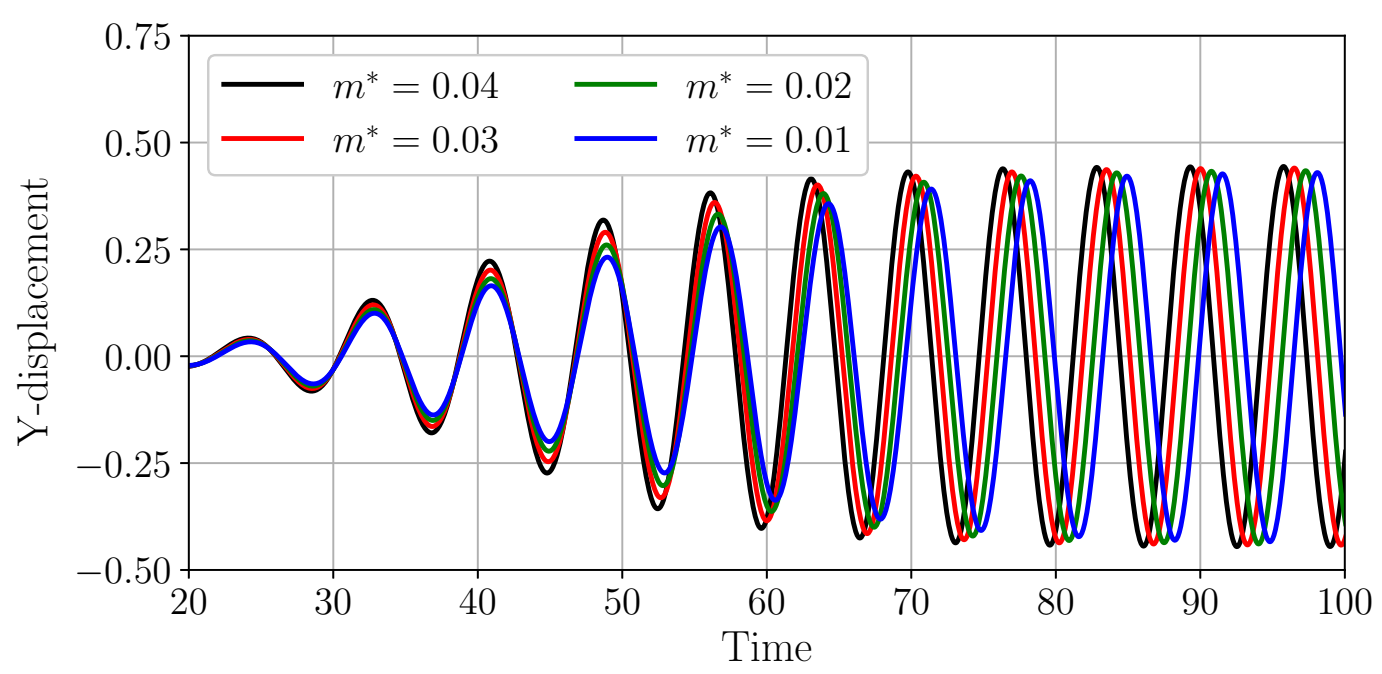

(b)

Figure 18: VIV of a circular cylinder: displacement response of the cylinder for four different values of $m^{*}$. (a) time range $[0,200]$ and (b) time range $[20,100]$.

\subsection{Rectangular body rising to the top of a fluid chamber}

As the last example, we consider the problem of rising of a lightweight rectangular block with rounded corners that was originally studied in Banks et al. [64]. The geometry and boundary conditions of the problem are shown schematically in Fig. 19. The density of the fluid is $\rho^{f}=1$, and its dynamic viscosity is $\mu^{f}=0.025$. The density of the solid block is $\rho_{b}=0.001$, which is $1 / 1000^{\text {th }}$ of that of the fluid. Due to the differences in the densities, the solid rises in the fluid under the influence of gravity. Following [64], the buoyancy on the solid is given as,

$$
F_{\text {buoyancy }}=-A_{b}\left[\rho_{b}-\rho^{f}\right] R(t),
$$

where, $A_{b}$ is the area of the solid and $R(t)$ is a ramp function, given by

$$
R(t)= \begin{cases}{[35+[-84+[70-20 t] t] t] t^{4}} & \text { if } 0 \leq t \leq 1 \\ 1, & \text { if } t>1\end{cases}
$$


For this example, the mass-ratio parameter is $m^{*}=0.001$. Such a significantly low value of $m^{*}$ poses serious difficulties in simulating this example using the conventional staggered scheme. In fact, the conventional staggered scheme without any relaxation parameters fails to simulate this particular problem. Banks et al. [64] report that the traditional partitioning algorithm for rigid bodies (TP-RB), see [63], requires an average of 85 sub-time-step iterations for each time step on the coarsest grid used in [64]. Banks et al. [64] successfully simulated this problem using their added-mass partition algorithm for rigid bodies (AMP-RB).

For the simulations, we consider two uniform meshes, as shown in Fig. 20. With each mesh, we perform the simulations with three different time steps, $\Delta t=\{0.02,0.01,0.005\}$. The fluid solver is the same as the one used for the circular cylinder example. The relaxation parameter for all the simulations is $\beta=0.0002$. The motion of the square body during its rise is plotted in terms of displacement and velocity response in Fig. 21 for all the six simulation cases, along with the reference solution from Banks et al. [64]. As shown in Fig. 21] there is no noticeable difference in the numerical results obtained with different meshes, and more importantly, the present results converge to the reference solution as the time step is reduced. Except for the initial spikes, the displacement response of the solid body is indistinguishable from the reference solution for $t \geq 3$. Typical contour plots of fluid pressure and plots of streamlines for time instants $t=2, t=4$ and $t=10$ are presented in Fig. 22. The present example demonstrates the excellent capability of this seemingly simple staggered scheme in computing accurate numerical solutions of very challenging FSI problems involving lightweight rigid solids.

We reiterate that the fluid and solid solvers do not require any additional information other than the standard Dirichlet and Neumann boundary conditions, respectively, for the fluid and solid sub-problems, from their counterparts.

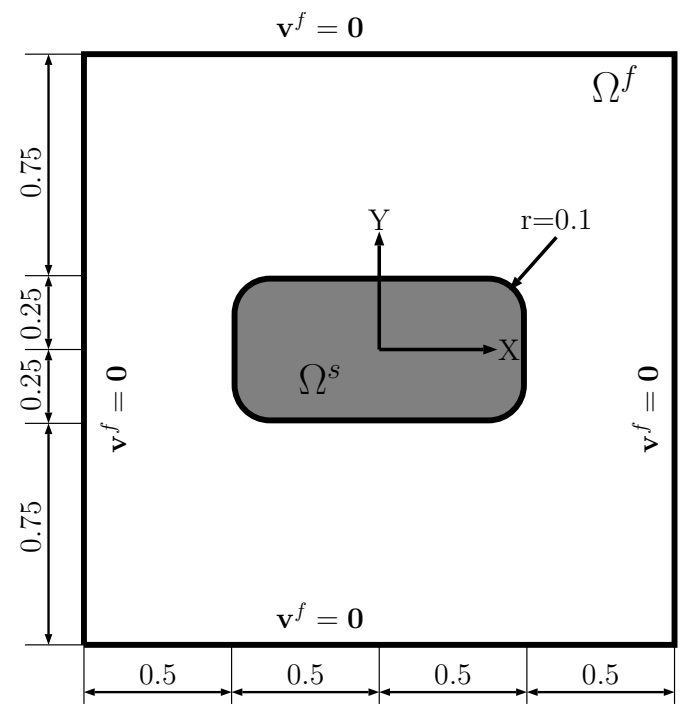

Figure 19: Rising rectangle: geometry and boundary conditions. 


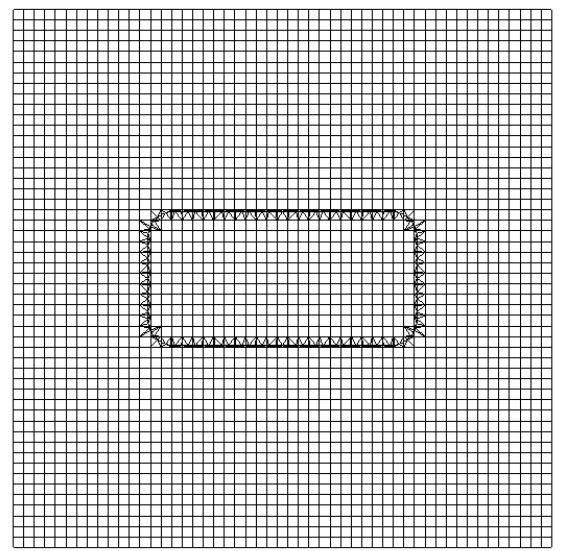

(a) Mesh $1(51 \times 51$ elements $)$

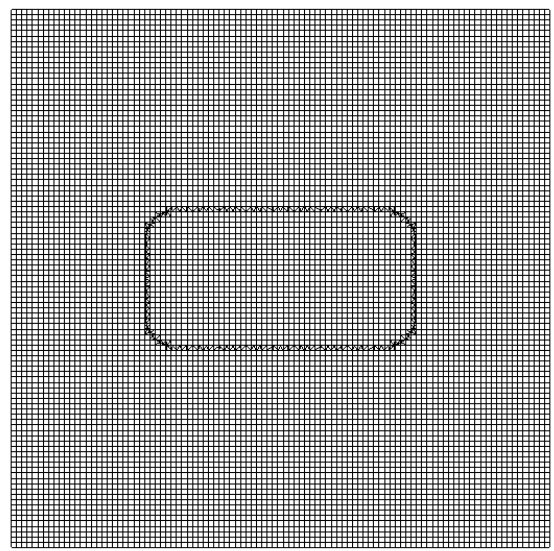

(b) Mesh $2(101 \times 101$ elements $)$

Figure 20: Rising rectangle: finite element meshes with the original position of the rectangle.

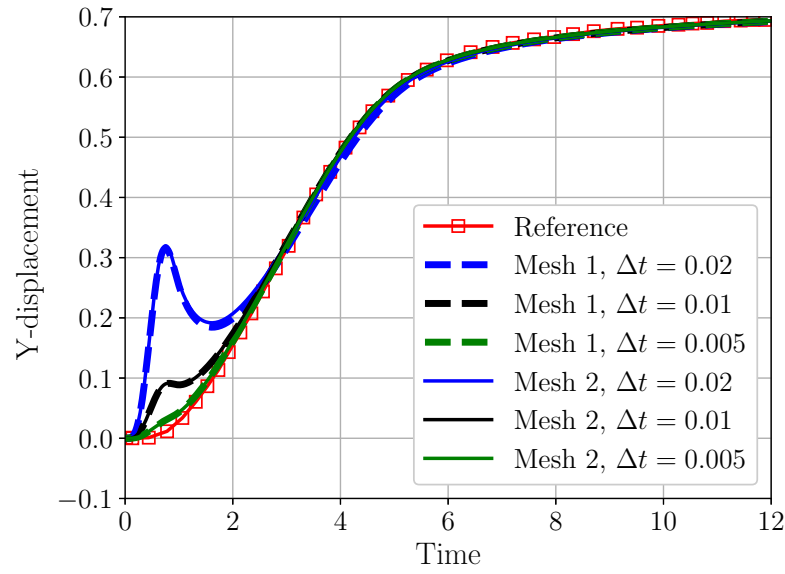

(a) Displacement response

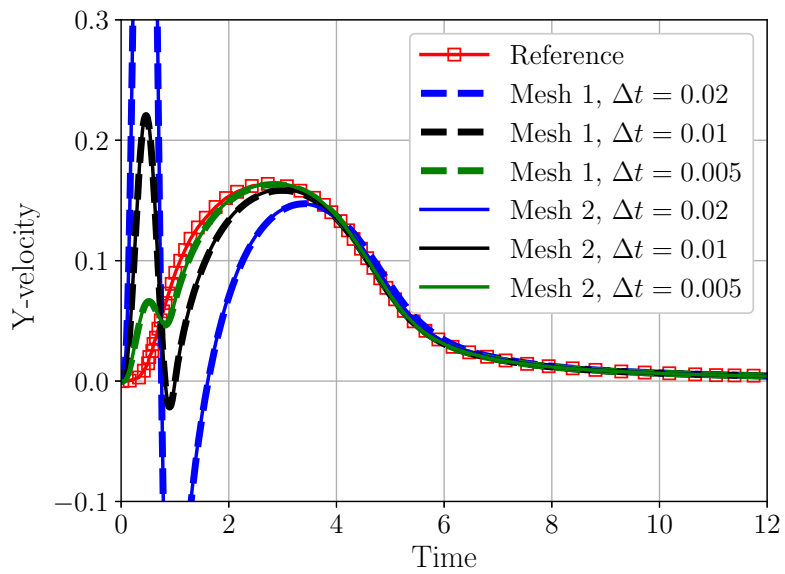

(b) Velocity response

Figure 21: Rising rectangle: displacement and velocity response of the solid body with M1 and M2 meshes and different time steps. The reference solution is from Banks et al. 64]. 


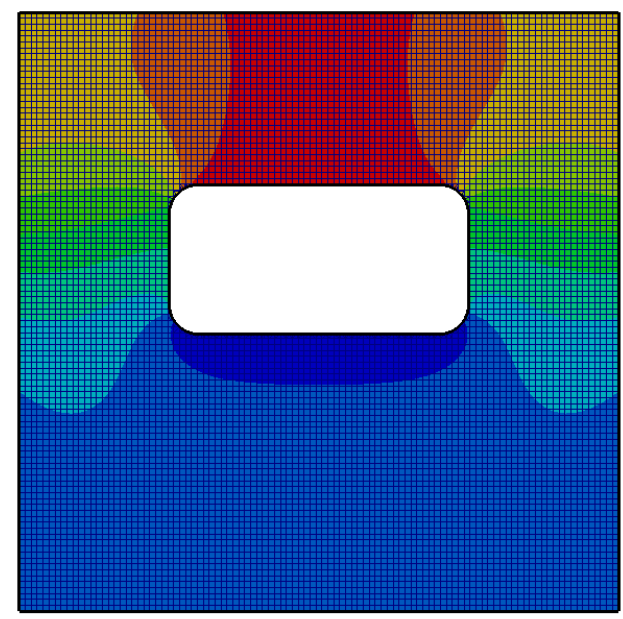

(a) $t=2.0$

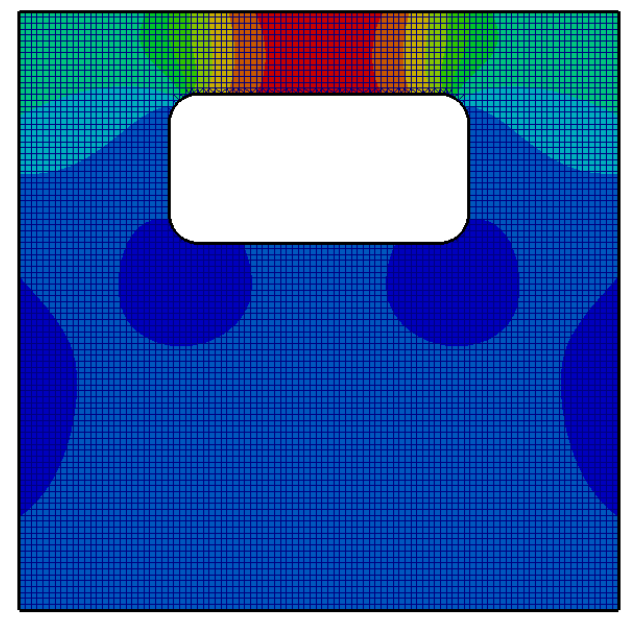

$$
\begin{aligned}
& \text { pressure } \\
& -0.35 \\
& -0.48 \\
& -0.60 \\
& -0.71 \\
& -0.94
\end{aligned}
$$

(c) $t=4.0$
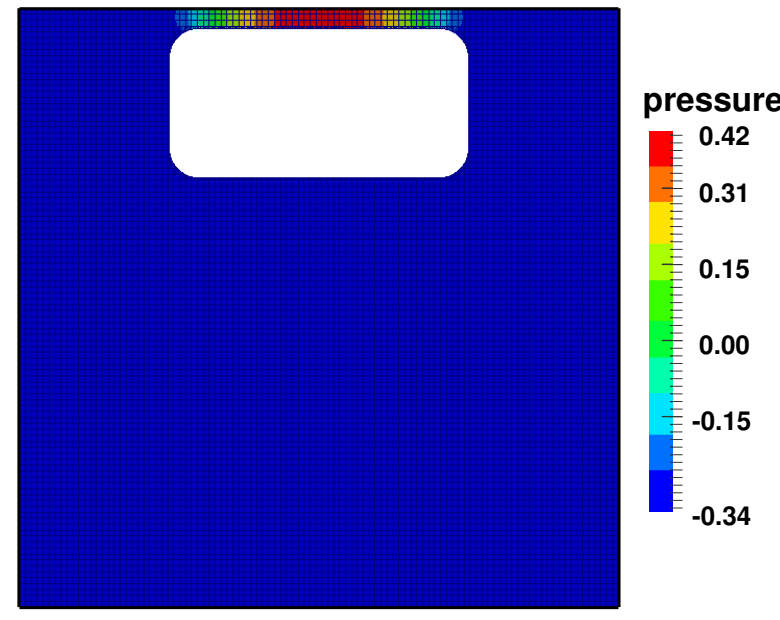

(e) $t=10.0$

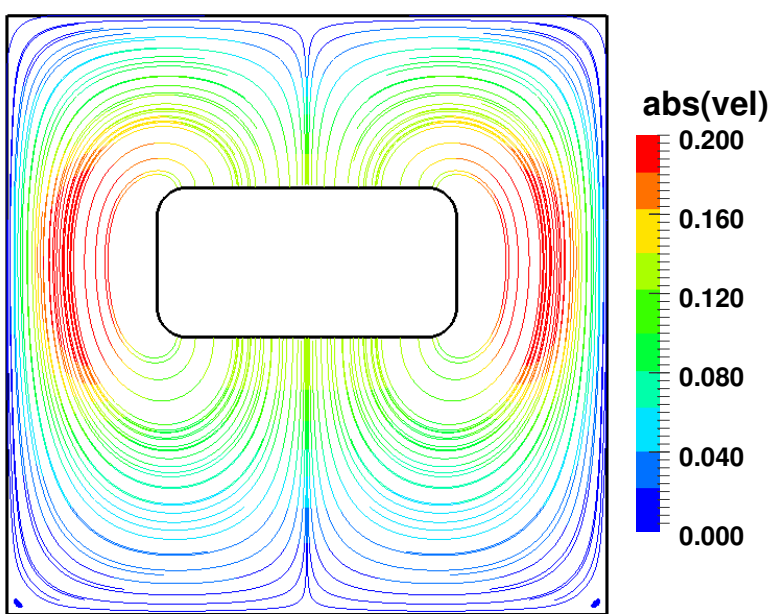

(b) $t=2.0$

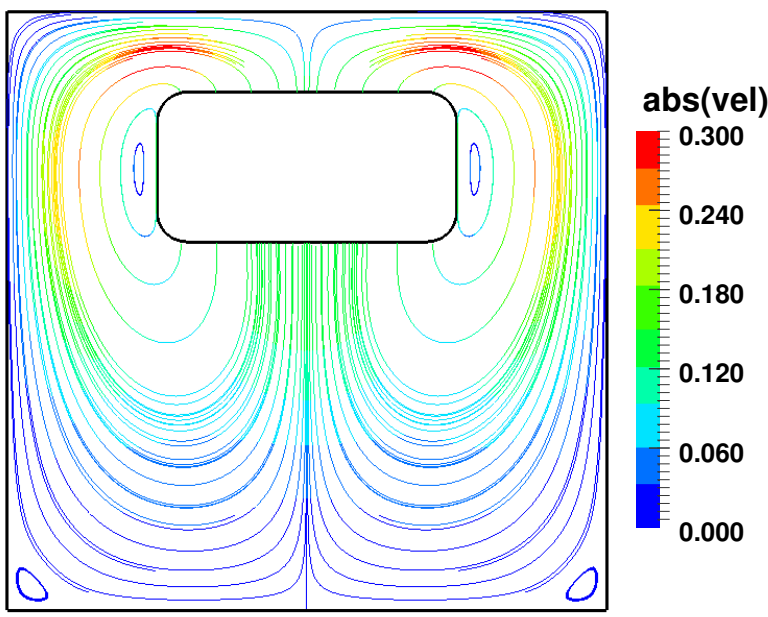

(d) $t=4.0$

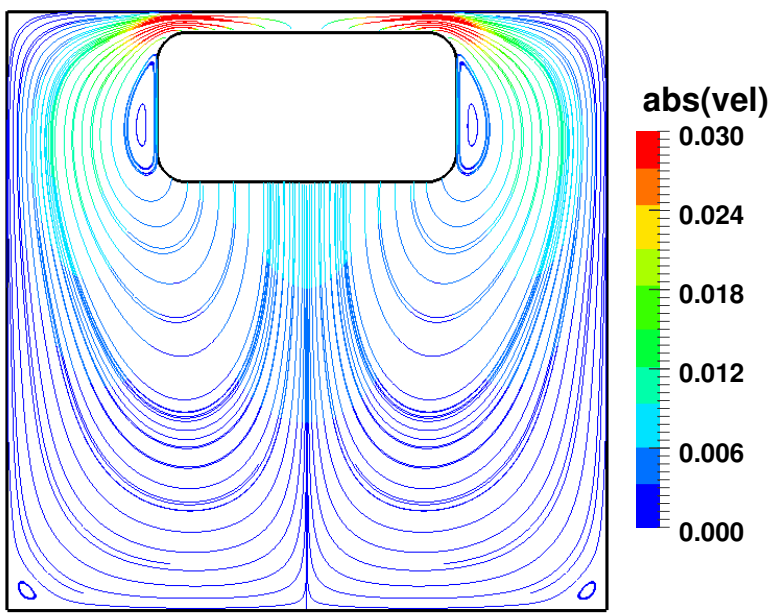

(f) $t=10.0$

Figure 22: Rising rectangle: contour plots of pressure and streamlines at three different time instants obtained with Mesh 2 and $\Delta t=0.01$. 


\section{Selection criterion for the relaxation parameter $(\beta)$}

Almost all of the staggered schemes rely on relaxation parameter(s) for their success. Such a parameter in the proposed scheme that needs to be supplied by the user is the relaxation parameter $\beta$. The minor inconvenience in selecting a suitable value for $\beta$ is negligible when compared with the remarkable accuracy of the computed numerical results as well as the computational efficiency of the staggered scheme. Moreover, the selection of $\beta$ is not completely arbitrary, as discussed below. We offer the following insights to help in choosing the value of $\beta$ for the given value of $m^{*}$.

(i) Considering the stability condition 31, obtained from the analysis of the linear model problem in Section 4.5 into account, the valid range of $\beta$ for any non-zero value of $m^{*}$ becomes,

$$
0<\beta \leq \alpha,
$$

for the worst-case scenario of zero numerical damping, i.e. $\rho_{\infty}^{s}=\rho_{\infty}^{f}=1$. While this stability condition might vary due to the spatial and temporal discretisation schemes for the fluid and solid solvers, the inclusion of physical damping and the manner in which boundary conditions are applied, our experience indicates that the variation is not by orders of magnitude for a particular value of $m^{*}$. Therefore, the above condition (35) serves as the starting point for choosing an appropriate value of $\beta$ for the given $m^{*}$.

(ii) The task of selecting $\beta$ is also eased by the fact that the simulation crashes quickly if the chosen value of $\beta$ is larger than that dictated by the stability limit; the farther the value of $\beta$ away from the stability limit the quicker the simulation crashes. It has been observed that the simulation crashes within the first 10-100 time steps, as illustrated in Fig. 23 for the example of galloping of a square for $m^{*}=0.2$ for different values of $\beta$. Thus, a value for $\beta$ that works can be selected relatively quickly, considering especially that the present staggered scheme is free from sub-iterations at every time step.

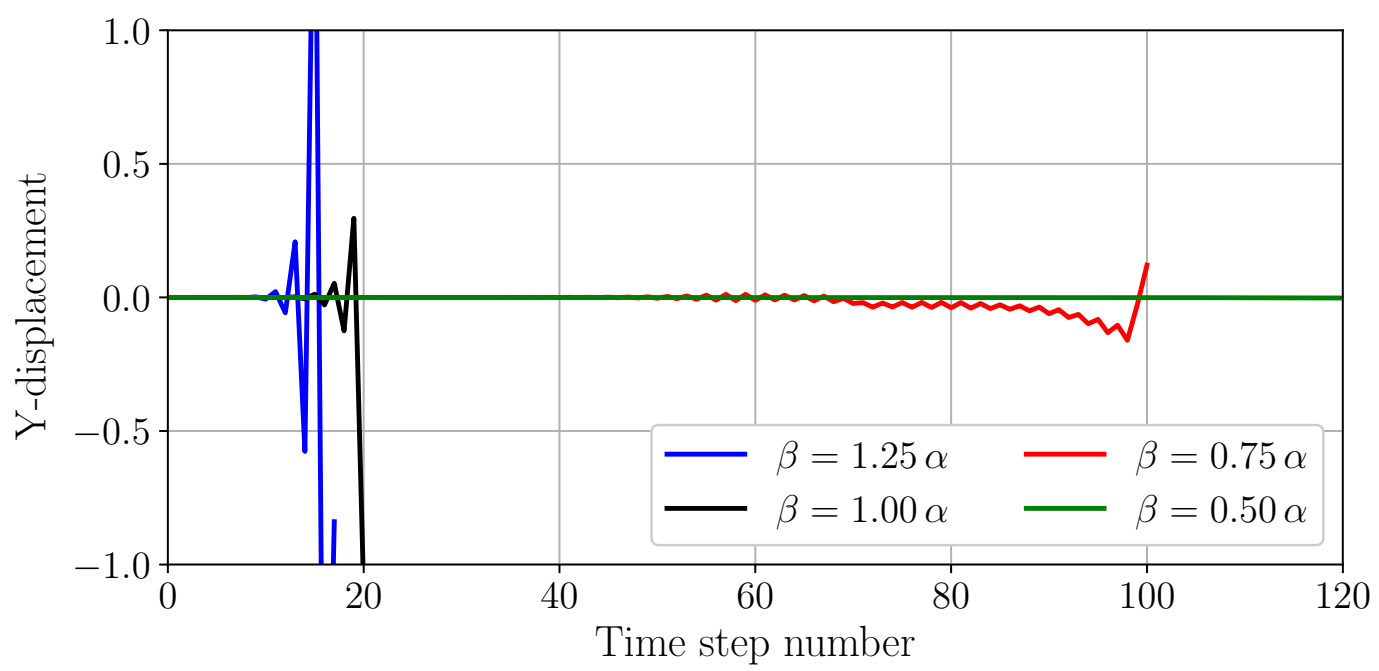

Figure 23: Sensitivity of the simulation crash with respect to $\beta$ for the example of galloping of square for $m^{*}=0.2$. 


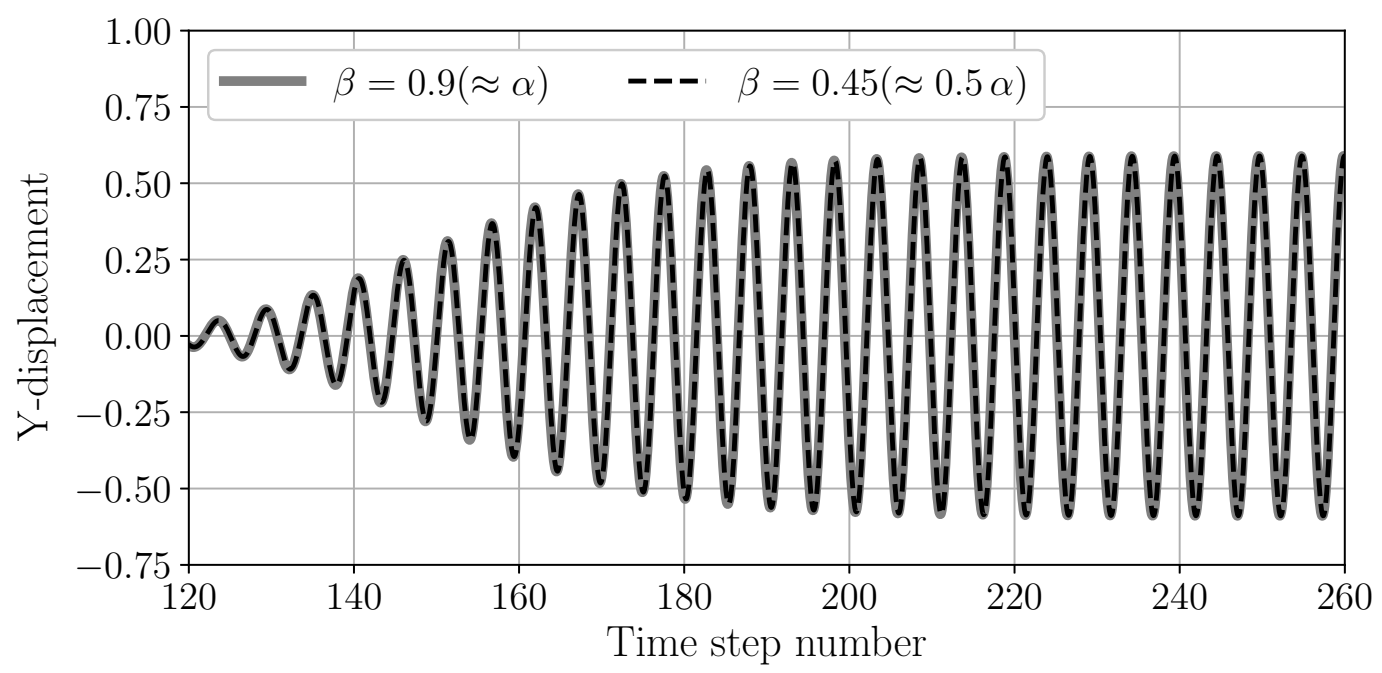

Figure 24: Displacement response of circular cylinder for $m^{*}=10$ for different values of $\beta$.

Based on our extensive experience in performing successful FSI simulations using the staggered scheme in the present work as well as the previous works [14, 15, 104],

$$
\text { the recommended value of } \beta \text { for } m^{*} \geq 0.01 \text { is } \beta=0.5 \alpha \text {. }
$$

The above recommendation is demonstrated to work with the example of galloping of a square for $m^{*}=0.2$ and $m^{*}=0.02$, and VIV of a circular cylinder for which $m^{*} \geq 0.01$. Further reduction of $\beta$ was found necessary only when $m^{*}$ is extremely small, as seen in the example of rising of the rectangular block. Since such extreme cases are uncommon in real-world applications, $\beta=0.5 \alpha$ is the recommended choice. This choice is further justified by the fact that it has a negligible effect on the accuracy of the results for the cases with large values of $m^{*}$, as demonstrated in Fig. 24 using the example of VIV of circular cylinder for $m^{*}=10$ with two different values of $\beta$.

Note: To further aid the curious reader in the numerical experiments, the following procedure is suggested for choosing a value of $\beta$ that works: Start with $\beta=\alpha$. If the simulation crashes, then reduce $\beta$ by $10 \%$ of $\alpha$ and repeat the simulation. With decrements of $10 \%$, the value of $\beta$ at the sixth increment is reduced to $0.5 \alpha$, which is the recommended value.

\section{Summary and Conclusions}

We have presented a second-order accurate staggered scheme for the simulation of fluid-structure interaction problems involving ultra-lightweight rigid solids. The novelty of the present work lies in the demonstrated abilities of the proposed scheme in performing accurate numerical simulations of fluid-rigid body interaction problems with significant added-mass using large time steps and without sub-iterations.

The capability of the staggered scheme to compute accurate solutions of FSI problems with significant added-mass is demonstrated with the example of transverse galloping of a square for $0.01 \leq m^{*} \leq 20$. The results obtained with the staggered scheme are shown to match remarkably well with those computed with the monolithic scheme. Later, the staggered scheme is applied to study the vortex-induced vibrations of a circular cylinder with two translational DOFs for $0.1 \leq m^{*} \leq 10$ and with only transverse DOF for $0.01 \leq m^{*} \leq 0.1$. Finally, the excellent accuracy of the staggered is illustrated with an even more challenging example of a rectangular block with $m^{*}=0.001$ rising in a liquid chamber.

The recommended value of the relaxation parameter is $\beta=0.5 \alpha$ for $m^{*} \geq 0.01$. Guidelines for choosing the relaxation parameter $(\beta)$ are also provided. The amount of effort required in selecting a value of $\beta$ that works is negligible when compared with the computational benefits that can be realised with the proposed scheme.

In addition to its excellent accuracy when using large time steps, especially for problems with significant added-mass, the standout feature of the proposed staggered scheme is its non-intrusive nature. The scheme does not require any additional information from the fluid and solid solvers except the interface velocity from 
the solid solver and the interface traction from the fluid solver. Therefore, it can be adapted to develop an FSI framework by combining different fluid and solid solvers in a non-intrusive manner by treating the solvers as black boxes. This aspect of the staggered scheme is illustrated using two different CFD solvers in this work.

The ongoing work is focussed at the extension of the proposed scheme for 3D problems as well as for problems with high Reynolds number flows. Towards this, wrappers for coupling ANSYS and OpenFOAM CFD solvers with an in-house rigid-body dynamics solver are being explored.

\section{ACKNOWLEDGEMENTS}

The author acknowledges the support of the Supercomputing Wales project, which is part-funded by the European Regional Development Fund (ERDF) via the Welsh Government.

\section{References}

\section{References}

[1] H. Bungartz and M. Schäfer, editors. Fluid-Structure Interaction: Modelling, Simulation, Optimisation. Lecture Notes in Computational Science and Engineering. Springer, 2006.

[2] H. Bungartz, M. Mehl, and M. Schäfer, editors. Fluid Structure Interaction II: Modelling, Simulation, Optimization. Lecture Notes in Computational Science and Engineering. Springer, 2010.

[3] Y. Bazilevs, K. Takizawa, and T. E. Tezduyar. Computational Fluid-Structure Interaction: Methods and Applications. Wiley, 2013.

[4] G. Hou, J. Wang, and A. Layton. Numerical Methods for Fluid-Structure Interaction - A Review. Communications in Computational Physics, 12:337-377, 2012.

[5] J. Donea, A. Huerta, J.-Ph. Ponhot, and A. Rodriguez-Ferran. Arbitrary Lagrangian-Eulerian Methods. In E. Stein, R. de Borst, and T. J. R. Hughes, editors, Encyclopedia of Computational Mechanics, volume 1 of Encyclopedia of Computational Mechanics. John Wiley \& Sons, 2004.

[6] T. Nomura and T. J. R. Hughes. An arbitrary Lagrangian-Eulerian finite element method for interaction of fluid and a rigid body. Computer Methods in Applied Mechanics and Engineering, 95:115-138, 1992.

[7] W. G. Dettmer and D. Perić. A fully implicit computational strategy for strongly coupled fluid-solid interaction. Archives of Computational Methods in Engineering, 14:205-247, 2007.

[8] A. A. Johnson and T. E. Tezduyar. Mesh update strategies in parallel finite element computations of flow problems with moving boundaries and interfaces. Computer Methods in Applied Mechanics and Engineering, 119:73-94, 1994.

[9] T. Tezduyar. Finite element methods for flow problems with moving boundaries and interfaces. Archives of Computational Methods in Engineering, 8:83-130, 2001.

[10] C. S. Peskin. The immersed boundary method. Acta Numerica, 11:479-517, 2002.

[11] W. G. Dettmer, C. Kadapa, and D. Perić. A stabilised immersed boundary method on hierarchical bspline grids. Computer Methods in Applied Mechanics and Engineering, 311:415-437, 2016.

[12] A. J. Gil, A. A. Carreño, J. Bonet, and O. Hassan. An enhanced immersed structural potential method for fluid-structure interaction. Journal of Computational Physics, 250:178-205, 2013.

[13] R. Ghias, R. Mittal, and H. Dong. A sharp interface immersed boundary method for compressible viscous flows. Journal of Computational Physics, 225:528-553, 2007.

[14] C. Kadapa, W. G. Dettmer, and D. Perić. A stabilised immersed boundary method on hierarchical b-spline grids for fluid-rigid body interaction with solid-solid contact. Computer Methods in Applied Mechanics and Engineering, 318:242-269, 2017. 
[15] C. Kadapa, W. G. Dettmer, and D. Perić. A stabilised immersed framework on hierarchical b-spline grids for fluid-flexible structure interaction with solid-solid contact. Computer Methods in Applied Mechanics and Engineering, 335:472-489, 2018.

[16] R. L. LeVeque and Z. Li. The immersed interface method for elliptic equations with discontinuous coefficients and singular sources. SIAM Journal on Numerical Analysis, 31:1019-1044, 1994.

[17] Z. Li and M. Lai. The immersed interface method for the Navier-Stokes equations with singular forces. Journal of Computational Physics, 171:822-842, 2001.

[18] R. Mittal and G. Iaccarino. Immersed boundary methods. Annual Review of Fluid Mechanics, 37:239$261,2005$.

[19] L. Zhang, A. Gerstenherger, X. Wang, and W. K. Liu. Immersed finite element method. Computer Methods in Applied Mechanics and Engineering, 193:2051-2067, 2004.

[20] Y. Wang, P. K. Jimack, and M. A. Walkley. A one-field monolithic fictitious domain method for fluidstructure interactions. Computer Methods in Applied Mechanics and Engineering, 317:1146-1168, 2017.

[21] A. Massing, M. Larson, A. Logg, and M. Rognes. A Nitsche-based cut finite element method for a fluidstructure interaction problem. Communications in Applied Mathematics and Computational Science, 10(2):97-120, 2015.

[22] B. Schott, C. Ager, and W. A. Wall. Monolithic cut finite element-based approaches for fluid-structure interaction. International Journal for Numerical Methods in Engineering, 119:757-796, 2019.

[23] E. Burman, S. Claus, P. Hansbo, M. G. Larson, and A. Massing. CutFEM: Discretizing geometry and partial differential equations. International Journal for Numerical Methods in Engineering, 104:472$501,2014$.

[24] F. P. T. Baaijens. A fictitious domain/mortar element method for fluid-structure interaction. International Journal for Numerical Methods in Fluids, 35:743-761, 2001.

[25] R. Glowinski, Pan T. W., and J. Périaux. A fictitious domain method for external incompressible viscous flow modeled by Navier-Stokes equations. Computer Methods in Applied Mechanics and Engineering, 112:133-148, 1994.

[26] R. Glowinski, T. W. Pan, T. I. Hesla, and D. D. Joseph. A distributed Lagrange multiplier/fictitious domain method for particulate flows. International Journal of Multiphase Flow, 25:755-794, 1999.

[27] C. Kadapa, W. G. Dettmer, and D. Perić. A fictitious domain/distributed Lagrange multiplier based fluidstructure interaction scheme with hierarchical B-Spline grids. Computer Methods in Applied Mechanics and Engineering, 301:1-27, 2016.

[28] W. G. Dettmer and D. Perić. A computational framework for fluid-rigid body interaction: Finite element formulation and applications. Computer Methods in Applied Mechanics and Engineering, 195:16331666, 2006.

[29] J. Degroote, K. J. Bathe, and J. Vierendeels. Performance of a new partitioned procedure versus a monolithic procedure in fluid-structure interaction. Computers and Structures, 87:793-801, 2009.

[30] C. Michler, S. Hulshoff, E. van Brummelen, and R. de Borst. A monolithic approach to fluid-structure interaction. Computers and Fluids, 33(5-6):839-848, 2004.

[31] J. Hron and S. Turek. A Monolithic FEM/Multigrid Solver for an ALE Formulation of Fluid-Structure Interaction with Applications in Biomechanics. In H. J. Bungartz and M. Schfer, editors, Fluid-Structure Interaction, volume 53 of Lecture Notes in Computational Science and Engineering, pages 146-170. Springer, Berlin, Heidelberg, 2006. 
[32] M. Heil, A. L. Hazel, and J. Boyle. Solvers for large-displacement fluid-structure interaction problems: segregated versus monolithic approaches. Computational Mechanics, 43:91-101, 2008.

[33] A. Robinson-Mosher, C. Schroeder, and R. Fedkiw. A symmetric positive definite formulation for monolithic fluid structure interaction. Journal of Computational Physics, 230:1547-1566, 2011.

[34] F. Gibou and C. Min. Efficient symmetric positive definite second-order accurate monolithic solver for fluid/solid interactions. Journal of Computational Physics, 231:3246-3263, 2012.

[35] C. E. Brennen. A review of added mass and fluid internal forces. Technical report, Naval Civil Engineering Laboratory, Port Heuneme, CA 93043, USA, 1982.

[36] P. Causin, J. F. Gerbeau, and F. Nobile. Added-mass effect in the design of partitioned algorithms for fluid-structure problems. Computer Methods in Applied Mechanics and Engineering, 194:4506-4527, 2005.

[37] C. Conca, A. Osses, and J. Planchard. Added mass and damping in fluid-structure interaction. Computer Methods in Applied Mechanics and Engineering, 146:387-405, 1997.

[38] C. Förster, W. A. Wall, and E. Ramm. Artificial added mass instabilities in sequential staggered coupling of nonlinear structures and incompressible viscous flows. Computer Methods in Applied Mechanics and Engineering, 196:1278-1293, 2007.

[39] E. H. van Brummelen. Added mass effects of compressible and incompressible flows in fluid-structure interaction. Journal of Applied Mechanics, 76:021206, 2009.

[40] C. A. Felippa, K. C. Park, and C. Farhat. Partitioned analysis of coupled mechanical systems. Computer Methods in Applied Mechanics and Engineering, 190:3247-3270, 2001.

[41] L. Florentie, D. S. Blom, T. P. Scholcz, A. H. van Zuijlen, and H. Bijl. Analysis of space mapping algorithms for application to partitioned fluid-structure interaction problems. International Journal for Numerical Methods in Engineering, 2016.

[42] W. G. Dettmer and D. Perić. A new staggered scheme for fluid-structure interaction. International Journal for Numerical Methods in Engineering, 93:1-22, 2013.

[43] M. Fernández and M. Moubachir. A Newton method using exact jacobians for solving fluid-structure coupling. Computers and Structures, 83:127-142, 2005.

[44] E. Burman and M. Fernández. Explicit strategies for incompressible fluid-structure interaction problems: Nitsche type mortaring versus Robin-Robin coupling. International Journal for Numerical Methods in Engineering, 97:739-758, 2014.

[45] E. Burman and M. A. Fernández. Stabilization of explicit coupling in fluid-structure interaction involving fluid incompressibility. Computer Methods in Applied Mechanics and Engineering, 198:766-784, 2009.

[46] N. D. dos Santos, J.-F. Gerbeau, and J.-F. Bourgat. A partitioned fluid-structure algorithm for elastic thin valves with contact. Computer Methods in Applied Mechanics and Engineering, 2008.

[47] M. Breuer, G. De Nayer, M. Münsch, Gallinger T., and R. Wüchner. Fluid-structure interaction using a partitioned semi-implicit predictor-corrector coupling scheme for the application of large-eddy simulation. Journal of Fluids and Structures, 29:107-130, 2012.

[48] S. Badia, F. Nobile, and C. Vergara. Fluid-structure partitioned procedures based on Robin transmission conditions. Journal of Computational Physics, 227:7027-7051, 2008.

[49] C. Wood, A. J. Gil, O. Hassan, and J. Bonet. Partitioned block-Gauss-Seidel coupling for dynamic fluid-structure interaction. Computers and Structures, 88:1367-1382, 2010. 
[50] H. Matthies and J. Steindorf. Partitioned but strongly coupled iteration schemes for nonlinear fluidstructure interaction. Computers and Structures, 2002.

[51] C. Farhat, K. G. van der Zee, and P. Geuzaine. Provably second-order time-accurate loosely-coupled solution algorithms for transient nonlinear computational aeroelasticity. Computer Methods in Applied Mechanics and Engineering, 195:1973-2001, 2006.

[52] G. Guidoboni, R. Glowinski, N. Cavallini, and S. Canic. Stable loosely-coupled-type algorithm for fluid-structure interaction in blood flow. Journal of Computational Physics, 228:6916-6937, 2009.

[53] R. Jaiman, P. Geubelle, E. Loth, and X. Jiao. Combined interface condition method for unsteady fluidstructure interaction. Computer Methods in Applied Mechanics and Engineering, 200:27-39, 2011.

[54] C. Michler, E. H. van Brummelen, S. J. Hulshoff, and R. de Borst. The relevance of conservation for stability and accuracy of numerical methods for fluid-structure interaction. Computer Methods in Applied Mechanics and Engineering, 192:4195-4215, 2003.

[55] E. H. van Brummelen. Partitioned iterative solution methods for fluid-structure interaction. International Journal for Numerical Methods in Fluids, 65:3-7, 2011.

[56] T. He, D. Zhou, and Y. Bao. Combined interface boundary condition method for fluid-rigid body interaction. Computer Methods in Applied Mechanics and Engineering, 223:81-102, 2012.

[57] T. He, D. Zhou, Z. Han, J. Tu, and J. Ma. Partitioned subiterative coupling schemes for aeroelasticity using combined interface boundary condition method. International Journal of Computational Fluid Dynamics, 28:272-300, 2014.

[58] T. He, and K. Zhang. An overview of the combined interface boundary condition method for fluidstructure interaction. Archives of Computational Methods in Engineering, 24:891-934, 2017.

[59] T. He, H. Zhang, and K. Zhang. A smoothed finite element approach for computational fluid dynamics: applications to incompressible flows and fluid-structure interaction, Computational Mechanics, 62:1037-1057, 2018.

[60] T. He, J. Yang, and C. Baniotopoulos. Improving the CBSbased partitioned semiimplicit coupling algorithm for fluid-structure interaction, International Journal for Numerical Methods in Fluids, 87:463-486, 2018.

[61] T. He. A partitioned implicit coupling strategy for incompressible flow past an oscillating cylinder, International Journal for Computational Methods, 12:1550012, 2015.

[62] S. R. Idelsohn, F. Del Pin, R. Rossi, and E. Oñate. Fluid-structure interaction problems with strong added-mass effect. International Journal for Numerical Methods in Engineering, 80:1261-1294, 2009.

[63] J. W. Banks, W. D. Henshaw, and D. W. Schwendeman. An analysis of a new stable partitioned algorithm for FSI problems. Part I: Incompressible flow and elastic solids. Journal of Computational Physics, 269:108-137, 2014.

[64] J. W. Banks, W. D. Henshaw, and D. W. Schwendeman. An analysisof a new stable partitioned algorithm for FSI problems. Part II: Incompressible flow and structural shells. Journal of Computational Physics, 268:399-416, 2014.

[65] M. Bukač, S. Čanić, R. Glowinski, B. Muha, and A. Quaini. A Modular, Operator Splitting Scheme for Fluid-Structure Interaction Problems with Thick Structures. International Journal for Numerical Methods in Fluids, 74:577-604, 2014.

[66] M. Bukač, S. Čanić, and B. Muha. A partitioned scheme for fluid-composite structure interaction problems. Journal of Computational Physics, 281:493-517, 2015. 
[67] A. Winterstein, C. Lerch, K. U. Bletzinger, and R. Wüchner. Partitioned simulation strategies for fluidstructure-control interaction problems by Gauss-Siedel formulations. Advanced Modeling and Simulation in Engineering Sciences, 5:29, 2018.

[68] R. K. Jaiman, N. R. Pillalamarri, and M. Z. Guan. A stable second-order partitioned iterative scheme for freely vibrating low-mass bluff bodies in a uniform flow. Computer Methods in Applied Mechanics and Engineering, 301:187-215, 2016.

[69] T He. Towards straightforward use of cell-based smoothed finite element method in fluid-structure interaction. Ocean Engineering, 157:350-363, 2018.

[70] W. A. Wall. Fluid-Struktur Interaktion mit stabilisierten Finiten Elementen. PhD thesis, University of Stuttgart, 1999.

[71] J. Sarrate, A. Huerta, and J. Donea. Arbitrary Lagrangian-Eulerian formulation for fluid-rigid body interaction. Computer Methods in Applied Mechanics and Engineering, 190:3171-3188, 2001.

[72] H.-J. Bungartz, F. Lindner, B. Gatzhammer, M. Mehl, K. Scheufele, A. Shukaev, and B. Uekermann. preCICE - A fully parallel library for multi-physics surface coupling. Computers and Fluids, 2016.

[73] M. Mehl, B. Uekermann, H. Bijl, D. Blom, B. Gatzhammer, and A. van Zuijlen. Parallel coupling numerics for partitioned fluid-structure interaction simulations. Computers \& Mathematics with Applications, 71(4):869-891, 2016.

[74] M. Neumann, S. Tiyyaguar, W. Wall, and E. Ramm. Robustness and efficiency aspects for computational fluid structure interaction. In E. Krause, Y. Shokin, M. Resch, and N. Shokina, editors, Computational Science and High Performance Computing II, volume 91 of Notes on Numerical Fluid Mechanics and Multidisciplinary Design, Berlin, Heidelberg, 2006. Springer.

[75] W. Zhang, Y. Jiang, and Z. Ye. Two better loosely coupled solution algorithms of CFD based aeroelastic simulation. Engineering Applications of Computational Fluid Mechanics, 1:253-262, 2007.

[76] T. Sarpkaya. A critical review of the intrinsic nature of vortex-induced vibrations. Journal of Fluids and Structures, 19:389-447, 2004.

[77] J. Chung and G. M. Hulbert. A family of single-step Houbolt time integration algorithms for structural dynamics. Computer Methods in Applied Mechanics and Engineering, 118:1-11, 1994.

[78] K. E. Jansen, C. H. Whiting, and G. M. Hulbert. A generalized- $\alpha$ method for integrating filtered NavierStokes equations with a stabilized finite element method. Computer Methods in Applied Mechanics and Engineering, 190:305-319, 2000.

[79] C. Kadapa, W. G. Dettmer, and D. Perić. On the advantages of using the first-order generalised-alpha scheme for structural dynamic problems. Computers and Structures, 193:226-238, 2017.

[80] N. M. Newmark. A method of computation for structural dynamics. Journal of the Engineering Mechanics Division (ASCE), 85:67-94, 1959.

[81] H. M. Hilber, T. J. R. Hughes, and R. L. Taylor. Improved numerical dissipation for time integration algorithms in structural dynamics. Earthquake Engineering and Structural Dynamics, 5:283-292, 1977.

[82] G. M. Hulbert and T. J. R. Hughes. An error analysis of truncated starting conditions in step-by-step time integration: consequences for structural dynamics. Earthquake Engineering and Structural Dynamics, 15:901-910, 1987.

[83] O. Alexsson. Iterative Solution Methods. Cambridge University Press, 1994.

[84] B. Bouscasse, A. Colagrossi, S. Marrone, and M. Antuono. Nonlinear-wave effects on fixed and floating bodies. Journal of Fluids and Structures, 42:112-129, 2013. 
[85] A. J. C. Crespo, C. Altomare, J. Dominguez, J. Gonzalez-Cao, and M. Gomez-Gesteria. Towards simulating floating offshore oscillating water column converters with Smoothed Particle Hydrodynamics. Coastal Engineering, 126:11-26, 2017.

[86] H. Bihs and A. Kamath. A combined level set/ghost cell immersed boundary representation for floating body simulations. International Journal for Numerical Methods in Fluids, 83:905-916, 2017.

[87] H. Gotoh and A. Khayyer. On the state-of-the-art of particle methods for coastal and ocean engineering. Coastal Engineering Journal, 60:79-103, 2018.

[88] J. P. Den Hartog. Mechanical Vibrations. New York, Toronto, London. Mc Graw-Hill Book Company, 4th edition, 1956.

[89] R. D. Blevins. Flow Induced Vibration. Van Nostrand Reinhold Company, New York, 1977.

[90] A. Sharma and V. Eswaran. Heat and fluid flow across a square cylinder in the two-dimensional laminar flow regime. Numerical Heat Transfer, Part A: Applications, 45:247-269, 2004.

[91] S. Sen, S. Mittal, and G. Biswas. Flow past a square cylinder at low Reynolds numbers. International Journal for Numerical Methods in Fluids, 67:1160-1174, 2011.

[92] A. K. Sahu, R. P. Chhabra, and V. Eswaran. Two-dimensional unsteady laminar flow of a power law fluid across a square cylinder. Journal of Non-Newtonian Fluid Mechanics, 160:157-167, 2009.

[93] A. Sohankar, C. Norberg, and L. Davidson. Low-Reynolds-number flow around a square cylinder at incidence: study of blockage, onset of vortex shedding and outlet boundary condition. International Journal for Numerical Methods in Fluids, 26:39-56, 1998.

[94] A. P. Singh, De A. K., V. K. Carpenter, V. Eswaran, and K. Muralidhar. Flow past a transversely oscillating square cylinder in free stream at low Reynolds numbers. International Journal for Numerical Methods in Fluids, 61:658-682, 2009.

[95] R. M. Darekar and S. J. Sherwin. Flow past a square-section cylinder with a wavy stagnation face. Journal of Fluid Mechanics, 426:263-295, 2001.

[96] M. Zhao, L. Cheng, and T. Zhou. Numerical simulation of vortex-induced vibration of a square cylinder at a low Reynolds number. Physics of Fluids, 25:023603, 2013.

[97] A. Sohankar, C. Norberg, and L. Davidson. Simulation of three-dimensional flow around a square cylinder at moderate Reynolds numbers. Physics of Fluids, 11:39-56, 1999.

[98] A. Joly, S. Etienne, and D. Pelletier. Galloping of square cylinders in cross-flow at low Reynolds numbers. Journal of Fluids and Structures, 28:232-243, 2012.

[99] I. Robertson, S. J. Sherwin, and P. W. Bearman. A numerical study of rotational and transverse galloping rectangular bodies. Journal of Fluids and Structures, 17:681-699, 2003.

[100] K. Sourav and S. Sen. Transition of VIV-only motion of a square cylinder to combined VIV and galloping at low Reynolds numbers. Ocean Engineering, 187:106208, 2019.

[101] J. Y. Shao, C. Shu, N. Y. Liu, and X. Zhao. Numerical investigation of vortex induced rotation of two square cylinders in tandem arrangement. Ocean Engineering, 171:485-495, 2019.

[102] S. Mittal and V. Kumar. Finite element study of vortex-induced cross-flow and in-line oscillations of a circular cylinder at low Reynolds numbers. International Journal for Numerical Methods in Fluids, 31:1087-1120, 1999.

[103] N. Jauvtis and C. H. K. Williamson. The effect of two degrees of freedom on vortex-induced vibration at low mass and damping. Journal of Fluid Mechanics, 509:23-62, 2004. 
[104] C. Kadapa, W. G. Dettmer, and D. Perić. Accurate iteration-free mixed-stabilised formulation for laminar incompressible Navier-Stokes: Applications to fluid-structure interaction. Journal of Fluids and Structures, 97:103077, 2020.

[105] R. K. Jaiman, M. Z. Guan, and T. P. Miyanawala. Partitioned iterative and dynamic subgrid-scale methods for freely vibrating square-section structures at subcritical Reynolds number. Computers and Fluids, 133:68-89, 2016.

[106] A. Yenduri, R. Ghoshal, R, and R. K. Jaiman. A new partitioned staggered scheme for flexible multibody interactions with strong inertial effects. Computer Methods in Applied Mechanics and Engineering, 315:316-347, 2017. 